University of Nebraska - Lincoln

DigitalCommons@University of Nebraska - Lincoln

Contemporary sexual selection does not explain variation in male display traits among populations

J. Colton Watts

Allissa Flynn

Brigitte Tenhumberg

Eileen Hebets

Follow this and additional works at: https://digitalcommons.unl.edu/bioscitenhumberg

Part of the Bioinformatics Commons, Computational Biology Commons, Other Animal Sciences Commons, and the Population Biology Commons

This Article is brought to you for free and open access by the Papers in the Biological Sciences at DigitalCommons@University of Nebraska - Lincoln. It has been accepted for inclusion in Brigitte Tenhumberg Papers by an authorized administrator of DigitalCommons@University of Nebraska - Lincoln. 


\title{
Contemporary sexual selection does not explain variation in male display traits among populations
}

\author{
J. Colton Watts, Allissa Flynn, \\ Brigitte Tenhumberg, and Eileen A. Hebets \\ School of Biological Sciences \\ University of Nebraska-Lincoln, Lincoln, Nebraska 68588 \\ Correspondence - J. C. Watts, email j.colton.watts@gmail.com
}

\begin{abstract}
Sexual selection is widely hypothesized to facilitate the evolution of reproductive isolation through divergence in sexual traits and sexual trait preferences among populations. However, direct evidence of divergent sexual selection causing intraspecific trait divergence remains limited. Using the wolf spider Schizocosa crassipes, we characterized patterns of female mate choice within and among geographic locations and related those patterns to geographic variation in male display traits to test whether divergent sexual selection caused by mate choice explains intraspecific trait variation. We found evidence of phenotypic selection on male behavior arising from female mate choice, but no evidence that selection varied among locations. Only those suites of morphological and behavioral traits that did not influence mate choice varied geographically. These results are inconsistent with ongoing divergent sexual selection underlying the observed intraspecific divergence in male display traits. These findings align with theory on the potentially restrictive conditions under which divergent sexual selection may persist, and suggest that
\end{abstract}

Published in Evolution 73:9 (September 2019), pp. 1927-1940.

doi:10.1111/evo.13808

Copyright (c) 2019 by the authors. Evolution copyright (c) The Society for the Study of

Evolution. Published by John Wiley Inc.

Submitted 15 February 2019; accepted 17 July 2019; published 25 July 2019. 
long-term studies capable of detecting periodic or transient divergent sexual selection will be critical to rigorously assess the relative importance of divergent sexual selection in intraspecific trait divergence.

Keywords: Divergence, mate choice, reproductive isolation, sexual selection, speciation

Sexual selection refers to the variation in reproductive success among genotypes that arises from differences in mating and fertilization success (Andersson 1994). It is widely implicated in the evolution of reproductive isolation and, as such, in the process of speciation (WestEberhard 1983; Panhuis et al. 2001; Schluter 2001; Turelli et al. 2001; Coyne and Orr 2004; Ritchie 2007; Safran et al. 2013). In particular, sexual selection can contribute to reproductive isolation by causing correlated changes in sexual traits and sexual trait preferences within a species that eventually lead to prezygotic isolation (West-Eberhard 1983; Panhuis et al. 2001). Such co-occurring changes in sexual traits and their associated preferences can arise from a variety of nonexclusive processes such as Fisherian runaway, ecological divergence, and reinforcement (reviewed in Schluter 2001; Safran et al. 2013).

It has become increasingly apparent, however, that these evolutionary and ecological processes (e.g., Fisherian runaway) may lead to intraspecific divergence in sexual traits and preferences only under certain, potentially restrictive conditions (Lande 1981; Higashi et al. 1999; Servedio and Noor 2003; Arnegard and Kondrashov 2004; Kirkpatrick and Nuismer 2004; van Doorn et al. 2004; Bolnick and Fitzpatrick 2007; Ritchie 2007; Weissing et al. 2011). Nevertheless, there is a wealth of evidence consistent with a role of sexual selection in the evolution of reproductive isolation and speciation (reviewed in Panhuis et al. 2001; Ritchie 2007). Most of this evidence consists of comparative studies demonstrating that taxa characterized by proxies of strong sexual selection (e.g., sexual dichromatism, courtship elaboration) contain greater numbers of species or show elevated rates of speciation (birds: Barraclough et al. 1995; Mitra et al. 1996; Møller and Cuervo 1998; Seddon et al. 2008; fish: Wagner et al. 2012; meta-analysis: Kraaijeveld et al. 2011). Other studies have demonstrated elevated rates of evolution in sexual traits with plausible links to prezygotic isolation relative to neutral markers (e.g., Masta and Maddison 2002) or traits involved in postzygotic isolation (e.g., Gleason 
and Ritchie 1998; Mendelson 2003). Still others have explicitly linked differences among species in sexual traits and sexual trait preferences to the action of sexual selection within species (e.g., Gray and Cade 2000; Boul et al. 2006).

In contrast to the abundance of data across distinct taxa that are consistent with the role of sexual selection in reproductive isolation, demonstrations of divergent sexual selection causing sexual trait divergence within a species remain relatively rare (fish: Endler and Houde 1995; Selz et al. 2016; arthropods: Svensson et al. 2006; Grace and Shaw 2012; birds: Wilkins et al. 2016). Thus, the abundance of macroevolutionary patterns suggestive of sexual selection's role in speciation contrasts sharply with a paucity of examples of the underlying microevolutionary process in action. In other words, we know relatively little about how (and how often) these evolutionary processes arise in nature, and how exactly these processes might lead to the divergence we currently observe at macroevolutionary scales.

Studying these underlying microevolutionary processes is essential to test our understanding of sexual selection's role in reproductive isolation and speciation. Demonstrating a role of sexual selection in intraspecific sexual trait divergence, however, requires (1) demonstrating ongoing sexual selection within a species, (2) establishing intraspecific differences in sexual selection (e.g., among populations), (3) determining whether those traits experiencing divergent sexual selection also vary within the species, and (4) testing whether sexual trait differences are correlated with divergent sexual trait preferences. Failure to support any one of these criteria would align with previously discussed theoretical constraints regarding processes underlying divergence in sexual traits and preferences. It might additionally suggest a role of other evolutionary processes (e.g., drift, natural selection) in causing intraspecific sexual trait divergence.

The wolf spider Schizocosa crassipes provides an ideal study system for assessing the role of divergent sexual selection in causing intraspecific divergence in sexual traits. Previous work on $S$. crassipes has demonstrated that the form of male courtship displays varies among localities in the southeastern United States (Miller et al. 1998). Schizocosa crassipes males not only produce a species-specific vibratory display, but also produce a visual display by waving their darkly pigmented, tufted first legs above their cephalothorax during courtship. Miller 
et al. (1998) demonstrated that the frequency distribution of different male display components (e.g., leg-waving behavior) varies among localities. Additionally, through reciprocal mating trials among a subset of localities, the authors suggested that variation in male display form could be sufficient to confer partial reproductive isolation. More recent cue isolation studies have confirmed that male displays affect mating success, as the successful transmission of the vibratory and visual display components have an additive effect on male copulation probability (Hebets and Uetz 1999; Stafstrom and Hebets 2013). Moreover, the experimental removal of male foreleg ornamentation (tufts) decreases male copulation probability, though this effect is contingent on the transmission of the vibratory signal (Stafstrom and Hebets 2013). However, whether standing variation in male display form affects mating success through female mate choice per se remains untested, as does the role of sexual selection caused by mate choice in explaining variation in male displays among localities.

In this study, we quantify sexual selection occurring through mate choice across a wide geographic range in $S$. crassipes including several of the locations studied by Miller et al. (1998). Our goal is to assess the extent to which sexual selection varies among localities and explains patterns of intraspecific trait variation. If divergent sexual selection has contributed to sexual trait divergence in $S$. crassipes, we would predict that (P1) females should mate nonrandomly with respect to male display traits (evidence of ongoing sexual selection), (P2) the relationship between male display traits and male mating success should differ among locations (intraspecific differences in sexual selection among locations), and (P3) display traits under differential phenotypic selection through mate choice should differ among locations. Provided that we find support for P1, P2, and P3, we then would also predict $\left(\mathrm{P}_{4}\right)$ covariation between sexual selection and sexual traits among localities. We test these predictions by first conducting mating trials to quantify phenotypic selection on male display traits (P1) and compare the estimated selection gradients among locations (P2). We then quantify differences in male display traits among localities and assess whether traits that differed among locations are those under selection ( $\left.\mathrm{P}_{3}\right)$ and test for significant correlations between sitespecific selection gradients and display trait values ( $\left.\mathrm{P}_{4}\right)$. 


\section{Methods}

\section{Collection and maintenance}

We collected individuals from seven locations comprising similar habitat in Mississippi and Florida, USA in 2016 and 2017. All location information, including location abbreviations used hereafter, is provided in Table 1. Four locations were sampled in both 2016 and 2017, while we were only able to collect individuals from the other three locations in either 2016 (LB and RS) or 2017 (LL). We collected all spiders within five days of each other in each year, and returned them to the laboratory within one week of collection. We subsequently moved the spiders to individual $5.8 \times 5.8 \times 7.6 \mathrm{~cm}$ plastic cages (AMAC Plastic Products Corp., Sausalito, CA). All spiders from a single collection location were moved to the cages on the same day, and we moved those spiders from different collection locations to cages on consecutive days to ensure that each group had equal amounts of time in vials during transit. Masking tape wrapped around cage prevented visual interaction between neighboring spiders during maintenance. We maintained the spiders on a standard diet of two small feeder crickets two to three times weekly and ad libitum water under a 12:12 LD cycle and constant temperature $\left(25^{\circ} \mathrm{C}\right)$. We checked individuals every other day for the presence of molt to determine the date of maturation.

Table 1. Geographic locations from which Schizocosa crassipes were collected in 2016 and/or 2017 for use in laboratory mating trials.

\begin{tabular}{|c|c|c|c|}
\hline \multicolumn{2}{|c|}{ Abbreviation } & \multirow{2}{*}{$\begin{array}{l}\begin{array}{l}\text { Location } \\
\text { (Latitude, Longitude) }\end{array} \\
31.4251,-90.9906\end{array}$} & \multirow{2}{*}{$\begin{array}{l}\text { Collection year(s) } \\
2016(n=31), 2017(n=5)\end{array}$} \\
\hline CSRA1 & Clear Springs Recreation Area Dam, Roxie, MS & & \\
\hline CSRA2 & Clear Springs Recreation Area Primitive Campground, Roxie, MS & $31.4377,-90.9828$ & $2016(n=22), 2017(n=21)$ \\
\hline LB & LeFleur's Bluff State Park, Jackson, MS & $32.3284,-90.1443$ & $2016(n=15)$ \\
\hline LL & Lake Lincoln State Park, Wesson, MS & $31.6781,-90.3348$ & $2017(n=21)$ \\
\hline NSP1 & Natchez State Park Primitive Camping Area, Stanton, MS & $31.6083,-91.2307$ & $2016(n=18), 2017(n=19)$ \\
\hline NSP2 & Natchez State Park Cabins, Stanton, MS & $31.5921,-91.2034$ & $2016(n=21), 2017(n=11)$ \\
\hline RS & River Styx, Evinston, FL & $29.5173,-82.2218$ & $2016(n=19)$ \\
\hline
\end{tabular}


We used individuals in mating trials once they reached approximately 14 days postmaturation, as this is the period in which females seem most receptive in other Schizocosa spp. (Uetz and Norton 2007). While it was possible to tightly control variation in female age, variation in male age was more difficult to control. First, Schizocosa are highly protandrous, making it difficult to obtain sufficient sample sizes with appropriately aged females without accepting some degree of variation in male age. Thus, in 2016, although all males and females used in mating trials were collected as subadults and, therefore, both female and male age and mating status were known, male age varied to a greater extent than female age (female age $=14.27 \pm 1.50$ days Mean \pm SD; male age $=17.92 \pm 7.83$ days Mean \pm SD). Moreover, because localities differ in the extent of protandry (J.C. Watts, unpubl. data), the degree to which it was necessary to accept a wider variation in male age differed among locations. This resulted in variation in male age among locations $\left(F_{5,120}=6.54, P<0.001\right)$. Males from CSRA2 and NSP2 were approximately eight days older on average than males from RS and CSRA1. Nevertheless, we found no association between male age and any of the three phenotypic trait components we used in our analyses (RC1: $F_{1,124}=0.23, P=0.630$; RC2: $F_{1,124}=0.25$, $P=0.617$ RC3: $\left.F_{1,124}=0.06, P=0.804\right)$, nor was male age associated with copulation success $\left(\chi_{1}^{2}=0.34, P=0.561\right)$. Thus, we did not include male age as a covariate in our subsequent analyses. In 2017, almost all males had matured by the time we arrived at the collection sites, so we were forced to collect mature males of unknown age and mating status. However, the high degree of protandry in $S$. crassipes allowed us to collect subadult females in 2017 to ensure their virginity and to control variation in female age, as we did in 2016. In 2017, the female age was $14.40 \pm 0.83$ (mean \pm SD). Female age did not differ among collection sites $\left(F_{6,195}=1.44, P=0.202\right)$.

\section{Mating trials}

We conducted all mating trials using randomly paired males and females from the same location. Consequently, our study did not evaluate whether any differences in traits and mate choice behavior cause behavioral isolation among spiders from different locations. Instead, our design allows us to characterize the effects of male traits 
on mating outcomes and compare those effects among locations. We gave each female and male one cricket 24 hours prior to mating trials to further standardize hunger state. Approximately 30 minutes prior to being used in a mating trial, we placed females on a $18.5^{-} \mathrm{cm}$ diameter filter paper (Whatman \#1 Qualitative Filter Paper) in an opaque plastic arena to collect female silk. Pheromones present in female wolf spider silk elicit male courtship behavior (Tietjen, 1977, 1979; Robertz and Uetz 2005). After collecting silk for 30 minutes, we moved females to a clear glass vial while the filter paper was inserted into an arena that facilitated video recordings from above and from the side of the spiders. The circumference of the bottom of the arena consisted of two stacked hoops of metal $(18.5 \mathrm{~cm}$ in diameter) between which each circular filter paper could be placed. Using three metal dowels as supports, we elevated the bottom of the arena so that the pheromone-laden filter paper constituted the substrate of the arena in each trial. We secured a clear acetate barrier to the outside of the metal hoop to create the walls of the arena. We left one half of the acetate barrier unobstructed to enable video recordings parallel to the plane of the arena substrate, while the other was covered with a printed photograph of leaf litter substrate taken from a site similar in substrate composition to those from which the study subjects were collected. We then placed the arena in a visually and vibrationally isolated chamber outfitted with two HD webcams (Logitech c290 HD Pro Webcam, Logitech, Fremont, CA) and a laser Doppler vibrometer (LDV) (Polytec PDV10o, Polytec, Irvine, CA). One webcam was located above the arena, perpendicular to the arena substrate, while the other was located to side, parallel to the arena substrate. The LDV was located above the arena so that the laser intersected the filter paper at a $90^{\circ}$ angle. The video and LDV recordings enabled us to score behavioral variables in greater detail than would be possible using live scoring, and the use of all three recording devices allowed us to cross-reference the three sources of information when scoring the mating trials.

Once the arena was prepared inside the recording chamber, we placed the female in the arena by upending the glass vial so that the female was in contact with the filter paper but remained sequestered under the vial. We then introduced the male to the arena in a similar fashion, where he remained sequestered for 1 minute to allow for acclimation to the arena. After 1 minute had elapsed, we released the 
male and allowed him to move freely about the arena and engage in courtship for 5 minutes. During this 5 -minute period, the female remained sequestered to ensure that all males were recorded for an equivalent 5-minute period without direct interactions with females. Preliminary observations suggested that allowing both individuals to freely interact from the outset of the trial drastically reduced the amount of courtship from which we could quantify male behavior, as females often chased males (preventing production of full courtship displays) or copulated within the first minute of the interaction.

Following the 5-minute period during which males, but not females, were free to move about the arena, we released the female and allowed the pair to interact freely for 30 minutes or until copulation occurred. We recorded whether the pair copulated (hereafter, copulation success) and the amount of that time elapsed between the release of the female and the copulation event (hereafter, latency to copulate). We conducted all trials between 1000 and 1900 hours and randomized their timing with respect to collection site to ensure that any differences in male behavior and female mate choice among locations were not caused by differences in the time of day.

\section{Quantifying male display traits}

\section{Morphological measurements}

We massed all individuals immediately prior to mating trials. Following mating trials, we euthanized males by freezing and subsequently photographed them before preserving them in 70\% ethanol. We retained all spiders as voucher specimens in our collection at UNL. In 2016, we photographed individuals using a SPOT Flex 15.2 64Mp camera (SPOT Imaging Solutions, Sterling Heights, MI) mounted to a stereo light microscope (Leica MZ16, Leica Microsystems, Bannockburn, IL). However, in 2017, the light microscope used in 2016 was unavailable. Consequently, we photographed individuals using a digital single lens reflex camera (Canon EOS Rebel T7i, Canon, Inc.) mounted with a $60 \mathrm{~mm} \mathrm{f/2.8} \mathrm{fixed} \mathrm{macro} \mathrm{lens} \mathrm{(Canon,} \mathrm{Inc.).} \mathrm{To} \mathrm{maximize} \mathrm{the}$ magnification of the photos taken with the macro photography setup, we took all photographs from the closest focal distance at which sharply focused images could be achieved (approximately $11 \mathrm{~cm}$ from 
the subject). In both years, we photographed all individuals in a single photography session to minimize variation in photography conditions. To further reduce variation in photography conditions, we took all photographs using manual camera settings. For each individual, we photographed the dorsal surface of the cephalothorax (the anterior body part) as well as the lateral surface of the male's right foreleg. We took all photographs against a laminated piece of white millimetric paper to allow calibration of subsequent measurements.

Using these photographs, we quantified each male's cephalothorax width, total brush area, and tibial darkness using ImageJ (version 1.49, National Institutes of Health, Bethesda, MA). We calculated cephalothorax width as the average of three measurements taken across the widest section of cephalothorax, perpendicular to the body's longitudinal axis. In contrast, we measured brush area only once for each male, as multiple measures of brush area were time prohibitive, and preliminary measurements showed little variation among repeated measurements. We also calculated tibial darkness as the average of three measures of the average grey value of the tibia, divided by the average grey value of four random selections of the white background to reduce any variation in darkness due to variation in illumination. Despite controlling photography conditions within years, the difference in photography methods between years resulted in systematic differences between years in tibial darkness measures $\left(F_{1,201}=1076.6\right.$, $P<0.001)$. We, thus, standardized tibial darkness measures to within-year means to control for systematic differences between years.

\section{Behavioral measurements}

Male $S$. crassipes courtship displays begin with the production of substrate-borne vibrations that appear to be produced via stridulation with the anterior-most pair of appendages, the pedipalps, and via tremulation of the abdomen Supporting Information Video). Males may produce vibrations for several minutes without interruption; during this time, they engage in periods of foreleg movement and percussive strikes (Miller et al. 1998). Foreleg movement consists of raising one or more of the four anterior-most legs (two on each side) above the substrate with the leg(s) either fully extended or curled to form a right angle (Miller et al. 1998). Percussive strikes (hereafter referred 
to as a "body bounces" or "bounces") consist of a rapid movement in which the body is forcefully slammed into the substrate (Miller et al. 1998). Although displays typically involve periods of stridulation and tremulation punctuated by leg movements and body bounces, many males produce one or several of these elements in isolation of the others.

We used several measures to capture variation among males in their courtship behavior (Table 2). We measured the latency (in seconds) to a male's first signal (visual or vibratory; hereafter, "latency to signal”) and to the first body bounce (latency to bounce). These measures presumably capture variation in a male's motivation to court. In particular, the latency to bounce provides a measure of how long a male took to produce a full bout of courtship, as not all bouts of signaling comprise the full courtship display typical of the species. We also calculated the number of body bounces performed per minute over the 5-minute observation window (bounce rate), as well as the total amount of time spent engaged in leg-waving behavior (total wave duration), which together provide measures of a male's overall courtship effort.

Table 2. Loadings of eight male phenotypic traits on three rotated components obtained from principal components analysis with varimax rotation.

\begin{tabular}{lccc} 
& $R C 1$ & $R C 2$ & $R C_{3}$ \\
& Courtship effort & Size/ornamentation & Leg wave duration \\
\hline Mass & 0.01 & $\mathbf{0 . 9 3}$ & -0.04 \\
CephWidth & -0.02 & $\mathbf{0 . 9 3}$ & 0.06 \\
BrushArea & 0.09 & $\mathbf{0 . 8 5}$ & 0.02 \\
TibialDark & 0.04 & -0.16 & -0.16 \\
LatencySig & $-\mathbf{0 . 7 7}$ & 0.04 & 0.12 \\
LatencyBounce & $\mathbf{- 0 . 8 4}$ & -0.06 & 0.04 \\
BounceRate & $\mathbf{0 . 7 8}$ & 0.10 & 0.34 \\
BounceInt & 0.01 & -0.09 & -0.40 \\
NoWaveBouts & $\mathbf{0 . 5 5}$ & 0.00 & $-\mathbf{0 . 6 8}$ \\
TotalWaveDur & $\mathbf{0 . 8 1}$ & 0.02 & 0.49 \\
MeanWaveDur & 0.36 & 0.01 & $\mathbf{0 . 8 7}$ \\
Prop. Variance & $\mathbf{0 . 2 7}$ & $\mathbf{0 . 2 3}$ & $\mathbf{0 . 1 6}$ \\
Cum. Variance & $\mathbf{0 . 2 7}$ & $\mathbf{0 . 5 0}$ & $\mathbf{0 . 6 6}$ \\
\hline
\end{tabular}

Axes are relabeled according to those phenotypic traits, which loaded most highly on the component.

Bolded values represent for each component those traits with loadings greater than 0.50 in absolute magnitude. 
We collected additional measures to quantify the form of signals produced by males while courting. We calculated the mean interval (in seconds) between consecutive body bounces, not including the period before the male's first bounce or the period after his last (bounce interval). We also calculated the total number of leg-waving bouts, where a leg-waving bout was delimited by a period of $1 \mathrm{~s}$ or greater in which neither of the male's forelegs were engaged in arching, extending/tapping, or waving (Miller et al. 1998). These measures reflect the rate of repetition of a signal element over a period of courtship. Because males often engaged in at least one of the leg-waving behaviors described by Miller et al. (1998) continuously (i.e., only producing a single, long bout of leg-waving during the observation period), we were unable to measure the mean interval between leg-waving bouts for all males. Instead, we recorded the mean duration of the leg-waving bouts (mean leg wave duration). For those males that produced only one leg-waving bout, this measure is equal to the total leg-wave duration.

We did not attempt to differentiate between different forms of legwaving movements as in Miller et al. (1998) because a single bout of leg-waving behavior comprises multiple rapid transitions from one form of movement to another, and consequently we were unable to reliably identify transition points. Moreover, we did not attempt to analyze variation in male vibratory signal amplitude, as we cannot account for variation in amplitude due to male position within the arena while signaling. We also did not attempt to analyze any frequency characteristics of the vibratory signal, as preliminary spectral analyses showed that the signal was highly broadband. We scored all recordings of mating trials using BORIS version 4.1.11 (Friard and Gamba, 2016), and we calculated the measures for each male using summary statistics available in BORIS.

\section{Reduction of phenotype space}

To simplify our analyses and account for correlations among traits, we performed principal components analysis (PCA) on the 11 phenotypic variables, applying a varimax rotation to increase the interpretability of the component loadings (Kaiser 1958). This resulted in three rotated components (RCs) collectively explaining 66\% of the variation in 
male phenotypic traits (Table 2 ). We did not retain additional components because their eigenvalues fell within the 95th percentile of those generated using parallel analysis (using the function hornpa() from the R package \{hornpa\} [Huang 2015]), suggesting that these additional eigenvalues mostly capture random noise (Horn 1965; O'Connor 2000).We conducted the PCA using the principal() function from the R package \{psych\} (Revelle 2017). To account for any effect of our decision to use PCA on our subsequent analyses, we also repeated these analyses using the 11 original phenotypic variables. We also considered analyses in which relative, rather than absolute, measures of male mass and tuft area were used. These alternative approaches did not affect our conclusions (see Supporting Information).

\section{Quantifying sexual selection}

To test the prediction that females should mate nonrandomly with respect to male display traits (P1), we began by estimating standardized directional selection gradients $(\beta)$ (Lande and Arnold 1983) for male phenotype RCs resulting from female mate choice. To accomplish this, we first $z$-standardized each male's scores for each phenotype RC by the mean and standard deviation of RC scores for males collected from the same location. Standardizing male RC scores by the local, rather than global, mean and standard deviation, places males' scores on a shared scale representing the variation in male phenotype that the local females with which they were paired would have been likely to encounter in the field. Then, we constructed a binomial logistic regression in which copulation success was modeled as a function of the linear combinations of RC scores. We used penalized logistic regressions (Firth 1993) using the function brglm() from the R package \{brglm\} (Kosmidis 2017) throughout our analyses to minimize issues associated with estimating regression parameters and their associated errors when successes or failures are rare among some combinations of predictor variables. We included collection year in all models to control for differences in copulation success across years. We assessed the significance of the estimated coefficient for each phenotype RC by comparing the full model to a model excluding the corresponding phenotype RC using a likelihood ratio test. We subsequently transformed the estimated logistic regression coefficients for each 
phenotype RC to obtain approximate selection gradients following Janzen and Stern (1998). We further transformed the approximate selection gradients and their associated standard errors by dividing each by the average predicted copulation probability so that the gradients represent changes in relative, not absolute, fitness with changing trait values (Janzen and Stern 1998).

To assess the support for differences in phenotypic selection on male phenotype RCs among collection locations (P2), we compared models describing potential forms of variation in selection among locations using an information-theoretic approach (Anderson and Burnham 2002; Anderson 2008). To allow for variation among locations in the associations between phenotype RCs and copulation success, we expanded our first-order multiple regression model (described above) to create a global model including an effect of collection site and interactions between collection site and each phenotype RC. The candidate model set included this global model as well as models comprising all possible combinations of the additional terms (i.e., collection site and interactions between collection site and phenotype RCs), taking care to respect marginality constraints associated with interactions terms. We also included the first-order multiple regression model to evaluate the support for models incorporating site-specific relationships relative to the initial, simpler model. We ranked the candidate models using Akaike's Information Criterion with correction for small sample size (AICc) and evaluated the relative support for each using Akaike weights and evidence ratios (i.e., the ratio of Akaike weight of a focal model to that of another model of interest) (Anderson 2008).

Quantifying geographic variation in male display traits

To test for differences in male phenotypes among locations, we performed an ANOVA on ranks for each RC separately, where ranked RC scores were modeled as a function of collection location. We used ANOVA on ranked scores to reduce any effects of RC score distributions on the ANOVA results. We included collection year in each model to control for differences in RC scores between years. We tested the significance of the collection location term for each RC using an $F$ test. To determine whether sexual traits under phenotypic selection through mate choice differ among locations (P3), we compared those 
RCs that differed among collection locations to those under phenotypic selection as identified by our selection gradient analysis (see Quantifying Sexual Selection section).We tested for covariation between sexual selection and sexual traits among localities (P4) using Pearson correlations of site-specific mean component scores and approximate selection gradients for each RC.

\section{Results}

\section{Quantifying male display traits}

We extracted three RCs that collectively explained $66 \%$ of male phenotypic variance (Table 2). The first RC (hereafter RC1) explained $27 \%$ of the overall variance and primarily reflected variables related to overall male courtship effort. Males that have higher RC1 scores have shorter latencies to the first signal and to the first body bounce, performed more body bounces and leg-waving bouts over the 5-minute observation period, and had a greater total duration of leg-waving behavior. The second RC (hereafter RC2) explained an additional 23\% of the male phenotypic variation. Males with larger RC2 scores had wider cephalothoraxes, greater body mass, and greater tibial brush area. The third rotated component (RC3), which accounts for another $16 \%$ of the phenotypic variation, represents residual variation in male legwaving behavior. A larger $\mathrm{RC}_{3}$ score indicates that, given the courtship effort of the male ( $\mathrm{RC} 1$ ), the male's leg-waving behavior consisted of fewer leg-waving bouts of greater mean duration, resulting in a greater total duration of leg waving. For clarity and ease of interpretation, we refer to these RCs according to those variables that loaded most strongly on them for the remainder of the paper (Table 2). Thus, we relabel RC1 as "courtship effort," RC2 as "size/ornamentation," and RC3 as "leg wave duration."

\section{Quantifying sexual selection}

In partial support of $\mathrm{P} 1$, we found significant effects of courtship effort $\left(\chi_{1}^{2}=9.46, P=0.002\right)$, but not size/ornamentation $\left(\chi_{1}^{2}=1.38\right.$, $P=0.240)$ or leg wave duration $\left(\chi_{1}^{2}=0.11, P=0.740\right)$, on male 

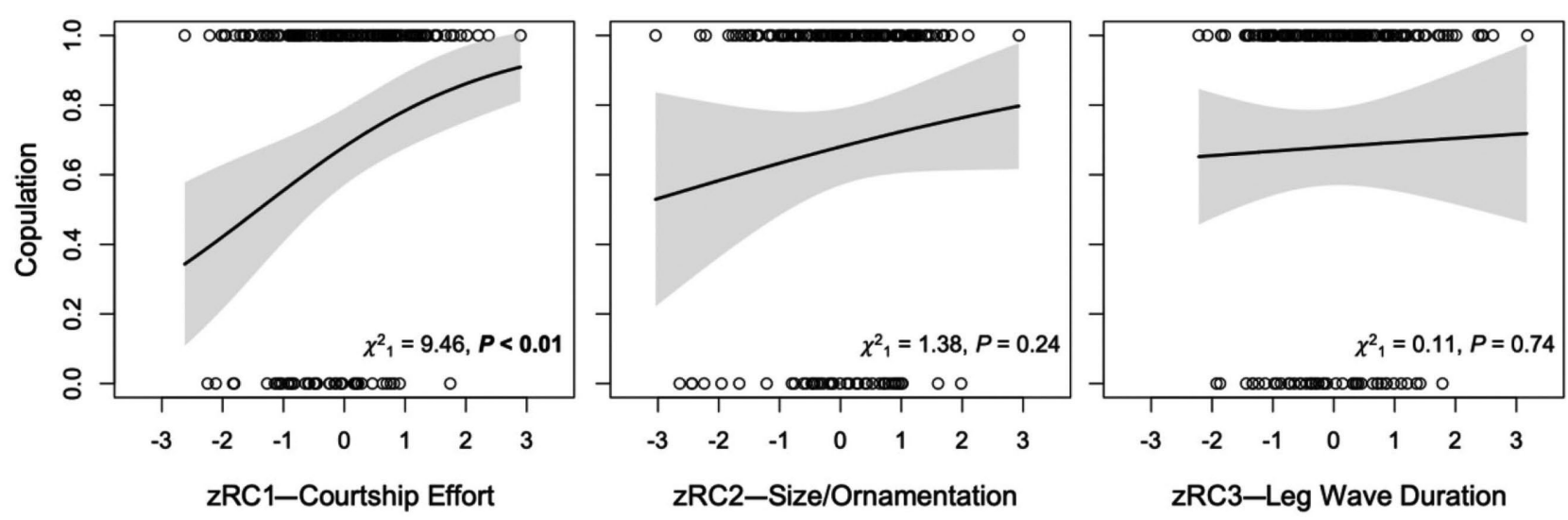

Figure 1. Relationships between $z$-score standardized rotated component (RC) scores representing male traits and copulation success. Each standardized RC is labeled according to the suite of traits, which loaded most strongly on it (see Table 2). Data are pooled across all localities sampled. Lines represent predictions from a penalized binomial logistic regression in which all rotated components were included. Shaded areas represent 95\% confidence intervals.

copulation success (overall model $\chi_{3}^{2}=11.16, P=0.011$ ) (Fig. 1). Males with greater courtship effort scores were more likely to copulate than males with lower courtship effort (estimate \pm std. error $=0.535 \pm$ 0.184 , approx. $\beta=0.086 \pm 0.030$, Fig. 1A). However, males with greater size/ornamentation scores were not significantly more likely to copulate than smaller, less ornamented males (estimate \pm std. error $=0.210 \pm 0.181$, approx. $\beta=0.034 \pm 0.029$, Fig. 1B). Similarly, males with greater leg wave duration scores were not significantly more likely to copulate (estimate \pm std. error $=0.057 \pm 0.176$, approx. $\beta$ $=0.009 \pm 0.028$, Fig. $1 \mathrm{C}$ ). Using the 11 original phenotypic variables and/or considering relative, rather than absolute, measures of male mass and tuft area did not change the interpretation of the data (see Supporting Information).

In contrast to P2, we found little support for statistical models including site-specific effects of the RCs representing male traits, though there was support for different intercepts among sites (Table 3). The best-supported model, which included site-specific intercepts, received about four times more support than the next best-supported model, which included no variation in slopes or intercepts of the relationships between male trait RCs and copulation success (evidence ratio $=$ 0.759/o.190 = 3.99) $($ Table 3). All other candidate models representing 
Table 3. Results of AICc model selection to evaluate support for differences among localities in the intercept or slope of the relationship between copulation success and each standardized rotated component $(\mathrm{RC})$ representing male traits.

\begin{tabular}{|c|c|c|c|c|c|c|c|c|}
\hline Site & $\begin{array}{l}\text { Courtship } \\
\text { effort:site }\end{array}$ & $\begin{array}{c}\text { Size/ } \\
\text { ornamentation:site }\end{array}$ & $\begin{array}{c}\text { Leg wave } \\
\text { duration:Site }\end{array}$ & $d f$ & LogLik & AICc & $\triangle A I C c$ & Weight \\
\hline \multirow[t]{2}{*}{+} & & & & 11 & -91.04 & 205.5 & 0.00 & 0.759 \\
\hline & & & & 5 & -98.97 & 208.2 & 2.78 & 0.190 \\
\hline+ & + & & & 17 & -87.35 & 212.0 & 6.54 & 0.029 \\
\hline+ & & + & & 17 & -87.76 & 212.8 & 7.36 & 0.019 \\
\hline+ & & & + & 17 & -89.78 & 216.9 & 11.41 & $<0.01$ \\
\hline+ & + & + & & 23 & -83.87 & 219.9 & 14.44 & $<0.01$ \\
\hline+ & + & & + & 23 & -86.72 & 225.6 & 20.13 & $<0.01$ \\
\hline+ & & + & + & 23 & -87.26 & 226.7 & 21.21 & $<0.01$ \\
\hline+ & + & + & + & 29 & -84.09 & 236.2 & 30.76 & $<0.01$ \\
\hline
\end{tabular}

Each row corresponds to candidate model, and + indicates the inclusion of a term in the model.

Each model also included an intercept and the linear effects of each rotated component (see Fig. 1 and text for details).

Standardized rotated components are referred to by the labels used in Table 2 and Figure 1.

possible forms of variation in phenotypic selection among localities received considerably less support than the model including no variation in slopes or intercepts (i.e., all evidence ratios_6 in support of the latter) (Table 3). Repeating the model selection procedure using the 11 original phenotypic variables and/or relative measures of male mass and tuft area produced similar model rankings (see Supporting Information).

For selection gradients estimated using a linear proxy of fitness as the response variable in the partial regression analysis, variation in the intercepts among sites would represent variation in female responsiveness, but not in the relative performance of different male phenotypes (Brooks and Endler 2001). However, because we use the binary outcome of copulation success as a fitness proxy, the change in fitness (i.e., the probability of copulating) with a unit increase in trait value depends on the probability of copulating for the present trait value. As a result, differences in intercepts (i.e., baseline copulation probabilities) among collection sites could correspond to differences in the approximate selection gradient (i.e., the average change in copulation probability with increasing trait value) over the same range of standardized component scores, despite a similar slope on the log-odds scale. Visualizing the changes in copulation probability with male trait values when accounting for differences in intercepts 

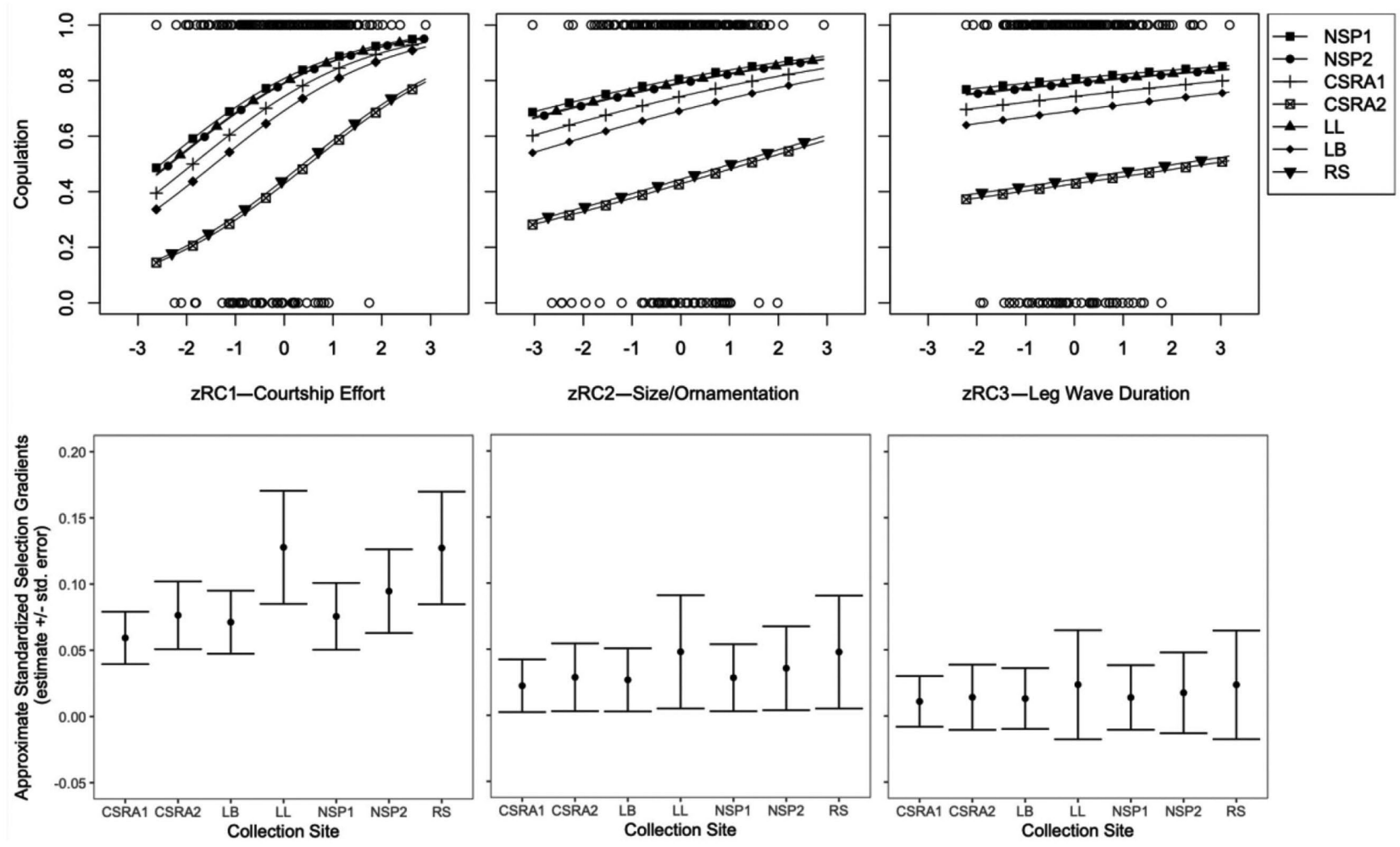

Figure 2. Top row: the effect of locality-specific intercepts on the relationships between copulation success and $z$-score standardized rotated component (RC) scores representing male traits. Lines represent predictions from the same penalized binomial logistic regression in Figure 1, but with an added term to allow intercepts to vary among collection sites (see Table 3). Predictions for different collection sites are denoted with jittered symbol types to aid in visualizing overlapping prediction lines. Confidence intervals are omitted to reduce visual clutter. Bottom row: Approximate selection gradients and associated standard errors for each rotated component (indicated in the top row) for each collection site. Approximate selection gradients were calculated using copulation probabilities for each male predicted by the penalized logistic regression model following Janzen and Sterns (1998).

suggests that variation in intercept among collection sites does not strongly affect the approximate selection gradient (Fig. 2). Moreover, calculating approximate selection gradients and their associated standard errors (Janzen and Stern 1998) for each site separately using predicted copulation probabilities from the model including site-specific intercepts revealed little evidence of among-site variation in selection on any of the three RCs representing male trait variation (Fig. 2). 

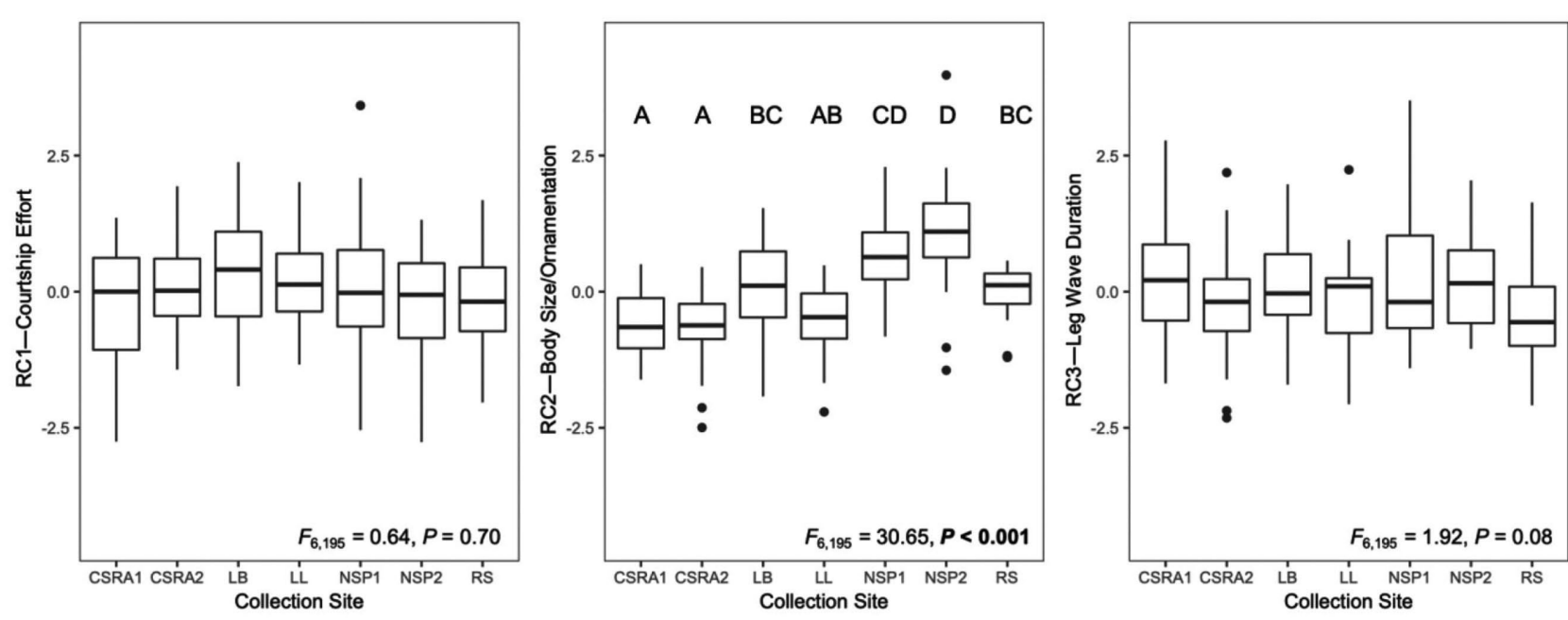

Figure 3. Geographic variation in rotated component (RC) scores representing male traits. Note that RC scores are not standardized to within-site means and standard deviations as in Figure 1 and Figure 2. Letter codes denote pairwise contrasts using Tukey's HSD post-hoc analysis; those sites not sharing a letter are significantly different.

\section{Quantifying geographic variation in male display traits}

After controlling for variation in RC scores between years, we found evidence of substantial geographic variation in suites of morphological, but not behavioral, traits. Collection sites did not differ in males' courtship effort scores $\left(F_{6,195}=0.641, P=0.700\right)$ (Fig. 3A), nor did we find an effect of collection year $\left(F_{1,195}=1.043, P=0.308\right)$. In contrast, males' size/ornamentation scores differed considerably among collection years $\left(F_{1,195}=10.115, P=0.002\right)$ and collection sites $\left(F_{6,195}=30.652, P<0.001\right)$. Males were generally larger and more ornamented in 2017 compared to 2016 (see Supporting Information). Most notably, spiders from the Clear Springs area of Mississippi (CSRA1 and CSRA2) differ strongly from those from the Natchez area (NSP1 and NSP2) approximately $30 \mathrm{~km}$ away (Supporting Information Fig. S1), while individuals from farther regions of Mississippi (LL, LB: $\sim 70-125 \mathrm{~km}$ ) and even Florida (RS: $\sim 900 \mathrm{~km}$ ) are intermediate in size/ornamentation scores with respect to these two groups (Fig. 3B). Leg wave duration scores also differed between collection years $\left(F_{1,195}=4.743, P=0.031\right)$, with individuals in 2017 having lower 
leg wave duration scores (see Supporting Information), while variation in leg wave duration scores among collection sites was marginally nonsignificant $\left(F_{6,195}=1.922, P=0.079\right)$ (Fig. $\left.3 \mathrm{C}\right)$. This trend was primarily driven by differences between the Florida collection site (RS) and individuals from CSRA1 and NSP2 (Fig. 3C), though neither contrast was significant after adjustment for family-wise error rate (RS-CSRA1: $P=0.148$; RS-NSP2: $P=0.181$ ). Thus, in contrast to our prediction that traits under selection through mate choice should differ among locations ( $\mathrm{P}_{3}$ ), only those suites of traits that did not influence mate choice varied among locations. This remains true even when considering all 11 original phenotypic variables (see Supporting Information). Moreover, for each $\mathrm{RC}$, the mean component scores and the site-specific approximate selection gradients were uncorrelated among collection sites (Pearson correlations, all $P>0.05$ ), inconsistent with $\mathrm{P}_{4}$.

\section{Discussion}

Using controlled laboratory mating trials, we failed to find evidence for a role of ongoing divergent sexual selection (operating through mate choice) in causing intraspecific variation in sexual traits among populations of the wolf spider $S$. crassipes. First, we found only partial support for our prediction that (P1) females should mate nonrandomly with respect to male display traits, generating ongoing sexual selection. Though we identified three RCs summarizing behavioral and morphological variation among male $S$. crassipes, only the RC representing a suite of variables related to courtship effort predicted male copulation success. Second, we found little support for variation in selection arising from mate choice among the localities we studied (contrary to P2). Even after calculating approximate selection gradients that account for differences in selection that arise due to geographic variation in baseline copulation probabilities and the distributions of male phenotype RC scores, there was little evidence for location-specific selection on male traits. Third, the only RCs that varied among locations were those that consistently failed to predict mating success, inconsistent with our prediction $\left(\mathrm{P}_{3}\right)$ that sexually selected traits should vary among locations. Moreover, approximate 
selection gradients and mean RC scores were uncorrelated among locations, contrary to the pattern that would be expected if ongoing divergent sexual selection underlies intraspecific trait divergence ( $\mathrm{P} 4)$. These conclusions hold even when conducting analyses using individual traits rather than $\mathrm{RC}$ scores and/or using relative measures of male mass and ornamentation.

The finding that male courtship effort best predicted mating success across all localities we sampled is generally unsurprising. Courtship effort (and in particular, courtship rate) is known to be one of the best predictors of mating success among Schizocosa wolf spiders (e.g., Delaney et al. 2007; Shamble et al. 2009; Hebets et al. 2011) as well as other spiders (e.g., Kotiaho et al. 1996). More generally, courtship rate influences copulation success in a wide variety of taxa, from beetles (Demary et al. 2006) and crickets (Wagner 1996) to tortoises (Galeotti et al. 2005) and birds (Collins et al. 1994), consistent with the hypothesis that females might generally evaluate males based on aspects of their motor performance (Byers et al. 2010). Interestingly, even those $S$. crassipes males with the lowest courtship effort scores were fairly likely to mate, resulting in generally weak (though statistically significant) selection.

In contrast to the effect of courtship effort, it is perhaps surprising that we found no association between copulation success and the component representing male size and ornamentation. Previous studies on $S$. crassipes have demonstrated that the experimental removal of tibial brushes decreases mating success, provided the vibratory courtship display is also detectable by the female (Stafstrom and Hebets 2013), and numerous studies implicate foreleg ornamentation per se in the mating success of the brush-legged congener S. ocreata (e.g., McClintock and Uetz 1996; Scheffer et al. 1996; Persons and Uetz 2005; Uetz and Norton 2007). The absence of any association between copulation success and variables related to size and ornamentation in our experiment suggests at least two possible explanations. First, in contrast to the presence versus absence of the brush (Stafstrom and Hebets 2013), standing variation in brush area may be insufficient to generate variation in female response. This might be expected if differential responses to the presence versus absence of brushes evolved after divergence from other Schizocosa to aid in species recognition but play no role in discriminating among conspecific 
males. This interpretation may be especially likely given that males of the morphological sister species to $S$. crassipes, Schizocosa floridana, do not develop tibial brushes upon maturation (Stratton 2005). Another nonmutually exclusive alternative is that the reduced mating success of males with shaved forelegs reported by Stafstrom and Hebets (2013) reflects changes in female responses as a result of an experimentally induced mismatch between body size and brush size. That is, females might in fact be perfectly inclined to accept a male with small brushes, provided the brushes adorn a correspondingly diminutive body. Shamble et al. (2009) made a similar argument to understand why females of another Schizocosa species, S. uetzi, preferred males with darker forelegs in playback experiments where other aspects of male phenotypes were standardized, but not in live mating trials where darker males were also larger and in better condition. Brush area covaries strongly with male size and body mass in our data set (see loadings in Table 2), providing much less opportunity for females to express such differential responses should they exist.

We also found no association between copulation success and the component representing variables related to leg-waving behavior. At first glance, this result might seem surprising given that previous work on S. crassipes (Hebets and Uetz 1999; Stafstrom and Hebets 2013) and other brush-legged Schizocosa (S. ocreata: Uetz et al. 2009, S. bilineata: E.A. Hebets unpubl. data) shows that visual signal transmission increases mating success. However, it is important to note that the PCA produces RCs that are by definition uncorrelated with one another. Thus, the RC corresponding to variables related to leg waving (i.e., RC3, see Table 2) contains only the variation in leg waving that does not overlap with variation in courtship effort (i.e., RC1). As a result, the lack of a statistical effect of the component representing leg-waving behavior does not imply that visual signaling is unimportant, but merely that any standing variation in visual signaling that affects copulation success could be largely correlated with variation in vibratory signaling (see loadings in Table 2). This is consistent with the hypothesis proposed by Stafstrom and Hebets (2013) that the sufficiency of both the visual and vibratory signal modalities for mating success allows them to function as backups and potentially convey overlapping information. While our study, like theirs, does not address the information content of these signals, our findings are 
consistent with their prediction that signals in these two modalities should be tightly correlated (Stafstrom and Hebets 2013).

In relation to our second prediction, we found little support for geographic variation in sexual selection arising from female mate choice. Among our candidate models to describe variation in selection among locations, there was some support for a model including different baseline copulation probabilities, but virtually no support for any other differences in selection among locations. Even our approximate selection gradients, which allow for differences in selection due to variation among collection sites in baseline copulation probabilities and the distributions of male phenotype scores, did not differ among sites for any of the three suites of male traits we considered. Given these results, we can conclude that the lack of selection on male size/ornamentation and leg wave duration is not due to variation among sites in selection on these suites of traits, but rather a consistent lack of association with copulation success among locations.

The geographic consistency in the approximate selection gradients for male courtship effort is striking. Although the locations we sampled are superficially similar in ecological conditions (e.g., substrate composition and presence of congeners), it may be surprising that location-specific patterns of female choice (and, thus, sexual selection) have not arisen due to neutral processes, such as drift, as the spatial extent of gene flow in wolf spiders appears to be quite limited (Reed et al. 2011). Thus, if patterns of gene flow in $S$. crassipes are similar to those of other wolf spiders, distance alone might be sufficient for divergence in sexual selection through differences in female mate choice. The geographic consistency we observe might be interpreted as evidence that female choice is under strong selection, preventing variation among locations in the relative mating success of different male phenotypes. However, we are not aware of any evidence that sexually selected traits correspond to any direct or indirect benefits (aside from any indirect benefits of producing preferred sons and choosy daughters) in Schizocosa wolf spiders. It is also possible that consistent selection on female mate choice among locations is generated by similar signaling environments (Guilford and Dawkins 1991; Endler 1992; Schluter and Price 1993). Indeed, the signaling environment has been hypothesized to be a primary factor shaping the evolution of Schizocosa sexual communication (e.g., Scheffer et al. 1996; 
Gibson and Uetz 2008; Hebets et al. 2008; Elias et al. 2010, Hebets et al. 2013). The superficial similarity in environments among the locations we studied could potentially facilitate consistent selection on female mate choice across the species range, though this remains to be tested.

Contrary to our third prediction, traits under phenotypic selection through mate choice did not differ among locations. In fact, the RC representing male courtship effort, which was the only component associated with copulation success, was also the only RC that did not show some variation among collection sites. Male size and ornamentation, which varied strongly among collection sites, was not associated with copulation success overall or in any of the sites we sampled. Similarly, there was a tendency for individual's leg wave duration scores to vary among collection sites, but this trait component also showed no correlation with mating success. Moreover, the variation among sites in the approximate selection gradient for each RC was uncorrelated with mean RC scores, contrary to $\mathrm{P} 4$.

These patterns are inconsistent with ongoing divergent sexual selection as the cause of intraspecific trait divergence in S. crassipes. A lack of ongoing divergent sexual selection aligns well with theoretical constraints on the conditions in which divergent sexual selection can occur (Lande 1981; Higashi et al. 1999; Servedio and Noor 2003; Arnegard and Kondrashov 2004; Kirkpatrick and Nuismer 2004; van Doorn et al. 2004; Bolnick and Fitzpatrick 2007; Ritchie 2007; Weissing et al. 2011). This does not, however, entirely rule out a role of divergent sexual selection in the intraspecific trait divergence we observed. It is possible that divergent sexual selection occurs only periodically within this species, such that we failed to detect it during the two consecutive breeding seasons in our study. Considerable temporal variation in sexual selection has been described in other study systems (Madsen and Shine 1993; Jann et al. 2000; Chaine and Lyon 2008; Kasumovic et al. 2008), though we know of no studies that demonstrate this in the context of spatially divergent sexual selection. It is also possible that sexual selection diverged within $S$. crassipes earlier in the history of these localities; if selection has since driven trait values to their local optima, selection on the remaining standing variation in trait values within localities may be negligible. A nonmutually exclusive explanation is that other evolutionary processes (e.g., drift, natural selection) 
are responsible for the intraspecific trait divergence we observed. If processes, such as drift or natural selection, have similar effects on the various aspects of male displays we quantified, the absence of intraspecific variation in male courtship effort might actually suggest that the contemporary consistency in sexual selection among localities constrains intraspecific trait divergence.

A historical role of divergent sexual selection may help to explain the contrast between the lack of selection on leg-waving behavior in our study and the demonstration in a previous study of partial reproductive isolation among localities that differ in leg-waving behavior (Miller et al. 1998), some of which were included in our study. If sexual selection on leg-waving behavior diverged among locations at some point in the past, it is possible that the evolutionary response in legwaving behavior might result in trait divergence (and partial reproductive isolation) among localities while rendering mating success relatively independent of the variation in leg-waving behavior within localities. Because we did not assay female responses to males from the other localities, our data cannot directly address this possibility. Nevertheless, the absence of ongoing divergent sexual selection suggests that reproductive isolation among these groups may not increase further unless other evolutionary processes (e.g., drift, natural selection) contribute to trait divergence in $S$. crassipes.

It is worth noting that we cannot rule out site-specific effects of traits on mating success that might occur in the natural setting but were masked in our controlled laboratory trials. Such effects might be particularly important given the hypothesized role of signal transmission and the signaling environment in sexual trait evolution in Schizocosa. Moreover, while previous studies have demonstrated the heritability of variation in male morphology and behavior both within and among species (Stratton and Uetz 1981; Fowler-Finn 2009),we cannot attribute the variation in male display traits we observed among collection sites to genetic differentiation. Thus, the intraspecific trait divergence we observed may instead reflect phenotypic plasticity, with lower levels of plasticity in the suite of traits that affects mating success.

In conclusion, our study demonstrates that contemporary patterns of sexual selection caused by mate choice do not explain intraspecific divergence in male display traits in $S$. crassipes. These findings 
contrast with a relatively limited number of empirical studies in other systems that demonstrate ongoing divergent sexual selection capable of explaining observed intraspecific trait differentiation (Endler and Houde 1995; Svensson et al. 2006; Grace and Shaw 2012; Selz et al. 2016; Wilkins et al. 2016). While our study reinforces previous concerns regarding our ability to infer the role of sexual selection within groups from patterns of sexual trait divergence among them (Panhuis et al. 2001; Ritchie 2007), it also emphasizes the potentially dynamic role of sexual selection in intraspecific trait divergence and reproductive isolation. Even in those cases, in which divergent sexual selection has caused sexual trait divergence, sexual selection may play such a role only transiently or do so periodically. Only longer-term studies that simultaneously quantify sexual selection within and among divergent groups will be able to identify the extent to which such transient or periodic dynamics are characteristic of sexual selection's role in divergence. Such studies will be critical to a rigorous understanding of the relative importance of sexual selection in causing intraspecific trait divergence and, ultimately, reproductive isolation and speciation.

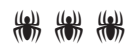

Author contributions JCW, BT, and EAH conceived the study. JCW collected specimens and conducted laboratory experiments. AF developed protocols for collecting experimental data and conducted the data collection. JCW conducted the statistical analyses and took the lead in writing the manuscript. All authors contributed to the writing and revision of the manuscript.

Acknowledgments We thank Cecile Renfro, Bailee Egan, Kelly Clay, Rowan McGinley, and Daniel Schoenberg for aiding with animal housing and maintenance. We thank William Wagner Jr., Matthew Wilkins, Daizaburo Shizuka, and John DeLong for insight into our experimental approach and analysis. Gail Stratton, Patricia Miller, and Kasey Fowler-Finn provided information on collection locations for our study organism. We thank Mississippi Wildlife, Fisheries, and Parks and the Mississippi Entomological Museum for facilitating our collections. This work was funded by a student research award from the Animal Behavior Society and by the Graduate Assistance in Areas of National Need program of the Department of Education.

Data archiving All data used in this publication are archived in the Dryad Digital Repository: https://doi.org/10.5061/dryad.gtv0342. 


\section{References}

Anderson, D. R. 2008. Model based inference in the life sciences: a primer on evidence. Springer Science and Business Media, New York, NY.

Anderson, D. R., and K. P. Burnham. 2002. Avoiding pitfalls when using information-theoretic methods. J. Wildl. Manage. 66:912-918.

Andersson, M. B. 1994. Sexual selection. Princeton Univ. Press, Princeton, NJ.

Arnegard, M. E., and A. S. Kondrashov. 2004. Sympatric speciation by sexual selection alone is unlikely. Evolution 58:222-237.

Barraclough, T. G., P. H. Harvey, and S. Nee. 1995. Sexual selection and taxonomic diversity in passerine birds. Proc. R. Soc. Lond. B 259:211-215.

Bolnick, D. I., and B.M. Fitzpatrick. 2007. Sympatric speciation: models and empirical evidence. Annu. Rev. Ecol. Evol. Syst. 38:459-487.

Boul, K. E., W. C. Funk, C. R. Darst, D. C. Cannatella, and M. J. Ryan. 20o6. Sexual selection drives speciation in an Amazonian frog. Proc. Roy. Soc. Lond. B. 274:399-406.

Brooks, R., and J. A. Endler. 2001. Female guppies agree to differ: phenotypic and genetic variation in mate-choice behavior and the consequences for sexual selection. Evolution 55:1644-1655.

Byers, J., E. A. Hebets, and J. Podos. 2010. Female mate choice based upon male motor performance. Anim. Behav. 79:771-778.

Chaine, A. S., and B. E. Lyon. 2008. Adaptive plasticity in female mate choice dampens sexual selection on male ornaments in the lark bunting. Science 319:459-462.

Collins, S. A., C. Hubbard, and A. M. Houtman. 1994. Female mate choice in the zebra finch-the effect of male beak colour and male song. Behav. Ecol. Sociobiol. 35:21-25.

Coyne, J. A., and H. A. Orr. 2004. Speciation. Sinauer, Sunderland, MA.

Delaney, K. J., J. A. Roberts, and G. W. Uetz. 2007. Male signaling behavior and sexual selection in a wolf spider (Araneae: Lycosidae): a test for dual functions. Behav. Ecol. Sociobiol. 62:67-75.

Demary, K., C. I. Michaelidis, and S. M. Lewis. 2006. Firefly courtship: behavioral and morphological predictors of male mating success in Photinus greeni. Ethology 112:485-492.

Elias, D. O., A. C. Mason, and E. A. Hebets. 2010. A signal-substrate match in the substrate-borne component of a multimodal courtship display. Curr. Zool. 56:370-378.

Endler, J. A. 1992. Signals, signal conditions, and the direction of evolution. Amer. Nat. 139:S125-S153.

Endler, J. A., and A. E. Houde. 1995. Geographic variation in female preferences for male traits in Poecilia reticulata. Evolution 49:456-468.

Firth, D. 1993. Bias reduction of maximum likelihood estimates. Biometrika 80:27-38. 
Fowler-Finn, K. D. 2009 Exploring the maintenance of and selection on two distinct male morphs in a Schizocosa wolf spider. Univ. of Nebraska, Lincoln, NE.

Friard, O., and M. Gamba. 2016. BORIS: a free, versatile open-source event logging software for video/audio coding and live observations. Methods Ecol. Evol. 7:1325-1330.

Galeotti, P., R. Sacchi, D. P. Rosa, and M. Fasola. 2005. Female preference for fastrate, high-pitched calls in Hermann's tortoises Testudo hermanni. Behav. Ecol. 16:301-308.

Gibson, J. S., and G.W. Uetz. 2008. Seismic communication and mate choice in wolf spiders: components of male seismic signals and mating success. Anim. Behav. 75:1253-1262.

Gleason, J. M., and M. G. Ritchie. 1998. Evolution of courtship song and reproductive isolation in the Drosophila willistoni species complex: do sexual signals diverge the most quickly? Evolution 52:1493-1500.

Grace, J. L., and K. L. Shaw. 2012. Incipient sexual isolation in Laupala cerasina: females discriminate population-level divergence in acoustic characters. Curr. Zool. 58:416-425.

Gray, D. A., and W. H. Cade. 200o. Sexual selection and speciation in field crickets. Proc. Natl. Acad. Sci. USA 97:14449-14454.

Guilford, T., and M. S. Dawkins. 1991. Receiver psychology and the evolution of animal signals. Anim. Behav. 42:1-14.

Hebets, E. A., and G.W. Uetz. 1999. Female responses to isolated signals from multimodal male courtship displays in the wolf spider genus Schizocosa (Araneae: Lycosidae). Anim. Behav. 57:865-872.

Hebets, E. A., D. O. Elias, A. C. Mason, G. L. Miller, and G. E. Stratton. 2008. Substrate-dependent signalling success in the wolf spider Schizocosa retrorsa. Anim. Behav. 75:605-615.

Hebets, E. A., J. A. Stafstrom, R. L. Rodriguez, and D. J. Wilgers. 2011. Enigmatic ornamentation eases male reliance on courtship performance for mating success. Anim. Behav. 81:963-972.

Hebets, E. A., C. J. Vink, L. Sullivan-Beckers, and M. F. Rosenthal. 2013. The dominance of seismic signaling and selection for signal complexity in Schizocosa multimodal courtship displays. Behav. Ecol. Sociobiol. 67:1483-1498.

Higashi, M., G. Takimoto, and N. Yamamura. 1999. Sympatric speciation by sexual selection. Nature 402:523-526.

Horn, J. L. 1965. A rationale and test for the number of factors in factor analysis. Psychometrika 30:179-185.

Huang, F. 2015. hornpa: Horn's (1965) test to determine the number of components/factors. R package version 1.o. Available at: https://CRAN.Rproject.org/package=hornpa

Jann, P., W. U. Blanckenhorn, and P. I. Ward. 2000. Temporal and microspatial variation in the intensities of natural and sexual selection in the yellow dung fly Scathophaga stercoraria. J. Evol. Biol. 13:927-938. 
Janzen, F. J., and H. S. Stern. 1998. Logistic regression for empirical studies of multivariate selection. Evolution 52:1564-1571.

Kaiser, H. F. 1958. The varimax criterion for analytic rotation in factor analysis. Psychometrika 23:187-200.

Kasumovic, M. M., M. J. Bruce, M. C. Andrade, and M. E. Herberstein. 2008. Spatial and temporal demographic variation drives within-season fluctuations in sexual selection. Evolution 62:2316-2325.

Kirkpatrick, M., and S. L. Nuismer. 2004. Sexual selection can constrain sympatric speciation. Proc. R. Soc. Lond. B. 271:687-693.

Kosmidis, I. 2017. brglm: bias reduction in binary-response generalized linear models. R package version o.6.1.

Kotiaho, J., R. V. Alatalo, J. Mappes, and S. Parri. 1996. Sexual selection in a wolf spider: male drumming activity, body size, and viability. Evolution 50:1977-1981.

Kraaijeveld, K., F. J. Kraaijeveld-Smit, and M. E. Maan. 2011. Sexual selection and speciation: the comparative evidence revisited. Biol. Rev. 86:367-377.

Lande, R. 1981. Models of speciation by sexual selection on polygenic traits. Proc. Natl. Acad. Sci. USA 78:3721-3725.

Lande, R., and S. J. Arnold. 1983. The measurement of selection on correlated characters. Evolution 37:1210-1226.

Madsen, T., and R. Shine. 1993. Temporal variability in sexual selection acting on reproductive tactics and body size in male snakes. Am. Nat. 141:167-171.

Masta, S. E., and W. P. Maddison. 2002. Sexual selection driving diversification in jumping spiders. Proc. Natl. Acad. Sci. USA 99:4442-4447.

McClintock, W. J., and G.W. Uetz. 1996. Female choice and pre-existing bias: visual cues during courtship in two Schizocosa wolf spiders (Araneae: Lycosidae). Anim. Behav. 52:167-181.

Mendelson, T. C. 2003. Sexual isolation evolves faster than hybrid inviability in a diverse and sexually dimorphic genus of fish (Percidae: Etheostoma). Evolution 57:317-327.

Miller, G. L., G. E. Stratton, P. R. Miller, and E. A. Hebets. 1998. Geographical variation in male courtship behaviour and sexual isolation in wolf spiders of the genus Schizocosa. Anim. Behav. 56:937-951.

Mitra, S., H. Landel, and S. Pruett-Jones. 1996. Species richness covaries with mating system in birds. Auk 113:544-551.

Møller, A. P., and J. J. Cuervo. 1998. Speciation and feather ornamentation in birds. Evolution 52:859-869.

O'Connor, B. P. 2000. SPSS and SAS programs for determining the number of components using parallel analysis and Velicer's MAP test. Behav. Res. Meth. Instrum. Comp. 32:396-402.

Panhuis, T. M., R. Butlin, M. Zuk, and T. Tregenza. 2001. Sexual selection and speciation. Trends Ecol. Evol. 16:364-371. 
Persons, M. H., and G. W. Uetz. 2005. Sexual cannibalism and mate choice decisions in wolf spiders: influence of male size and secondary sexual characters. Anim. Behav. 69:83-94.

Reed, D. H., V. H. Teoh, G. E. Stratton and R. A. Hataway. 2011. Levels of gene flow among populations of a wolf spider in a recently fragmented habitat: current versus historical rates. Conserv. Genet. 12:331-335.

Revelle, W. 2017. psych: procedures for psychological, psychometric, and personality research. R package version 1.7.5. Available at: https://CRAN.Rproject.org/package $=$ psych

Ritchie, M. G. 2007. Sexual selection and speciation. Annu. Rev. Ecol. Evol. Syst. 38:79-102.

Roberts, J. A., and G.W. Uetz. 2005. Information content of female chemical signals in the wolf spider, Schizocosa ocreata: male discrimination of reproductive state and receptivity. Anim. Behav. 70:217-223.

Safran, R. J., E. S. Scordato, L. B. Symes, R. L. Rodríguez, and T. C. Mendelson. 2013. Contributions of natural and sexual selection to the evolution of premating reproductive isolation: a research agenda. Trends Ecol. Evol. 28:643-650.

Scheffer, S. J., G. W. Uetz, and G. E. Stratton. 1996. Sexual selection, male morphology, and the efficacy of courtship signalling in two wolf spiders (Araneae: Lycosidae). Behav. Ecol. Sociobiol. 38:17-23.

Schluter, D. 2001. Ecology and the origin of species. Trends Ecol. Evol. 16:372-380.

Schluter, D., and T. Price. 1993. Honesty, perception and population divergence in sexually selected traits. Proc. R. Soc. Lond. B 253:117-122.

Seddon, N., R. M. Merrill, and J. A. Tobias. 2008. Sexually selected traits predict patterns of species richness in a diverse clade of suboscine birds. Am. Nat. 171:620-631.

Selz, O. M., R. Thommen, M. E. R. Pierotti, J. M. Anaya-Rojas, and O. Seehausen. 2016. Differences in male coloration are predicted by divergent sexual selection between populations of a cichlid fish. Proc. R. Soc. B. 283:20160172.

Servedio, M. R. and M. A. Noor. 2003. The role of reinforcement in speciation: theory and data. Ann. Rev. Ecol. Evol. Syst. 34:339-364.

Shamble, P. S., D. J. Wilgers, K. A. Swoboda and E. A. Hebets. 2009. Courtship effort is a better predictor of mating success than ornamentation for male wolf spiders. Behav. Ecol. 20:1242-1251.

Stafstrom, J. A. and E. A. Hebets. 2013. Female mate choice for multimodal courtship and the importance of the signaling background for selection on male ornamentation. Curr. Zool. 59:200-209.

Stratton, G. E. 2005. Evolution of ornamentation and courtship behavior in Schizocosa: insights from a phylogeny based on morphology (Araneae, Lycosidae). J. Arachnol. 33:347-376.

Stratton, G. E. and G. W. Uetz. 1981. Acoustic communication and reproductive isolation in two species of wolf spiders. Science 214:575-577. 
Svensson, E. I., F. Eroukhmanoff, and M. Friberg. 2006. Effects of natural and sexual selection on adaptive population divergence and premating isolation in a damselfly. Evolution 60:1242-1253.

Tietjen, W. J. 1977. Dragline-following by male lycosid spiders. Psyche 84:165-178.

- 1979. Is the sex pheromone of Lycosa rabida (Araneae:Lycosidae) deposited on a substratum? J. Arachnol. 6:207-212.

Turelli, M., N. H. Barton, and J. A. Coyne. 2001. Theory and speciation. Trends Ecol. Evol. 16:330-343.

Uetz, G. W. and S. Norton. 2007. Preference for male traits in female wolf spiders varies with the choice of available males, female age and reproductive state. Behav. Ecol. Sociobiol. 61:631-641.

Uetz, G.W., J.A. Roberts, and P.W. Taylor. 20o9. Multimodal communication and mate choice in wolf spiders: female response to multimodal versus unimodal signals. Anim. Behav. 78:299-305. van Doorn, G. S., U. Dieckmann, and F. J. Weissing. 2004. Sympatric speciation by sexual selection: a critical reevaluation. Am. Nat. 163:709-725.

Wagner, W. E. Jr. 1996. Convergent song preferences between female field crickets and acoustically orienting parasitoid flies. Behav. Ecol. 7:279-285.

Wagner, C. E., L. J. Harmon, and O. Seehausen. 2012. Ecological opportunity and sexual selection together predict adaptive radiation. Nature 487:366-369.

Weissing, F. J., P. Edelaar, and G. S. Van Doorn. 2011. Adaptive speciation theory: a conceptual review. Behav. Ecol. Sociobiol. 65:461-480.

West-Eberhard, M. J. 1983. Sexual selection, social competition, and speciation. Q. Rev. Biol. 58:155-183.

Wilkins, M. R., H. Karaardıç, Y. Vortman, T. L. Parchman, T. Albrecht, A. Petrželková, L. Özkan, P. L. Pap, J. K. Hubbard, A. K. Hund, et al. 2016. Phenotypic differentiation is associated with divergent sexual selection among closely related barn swallow populations. J. Evol. Biol. 29:2410- 2421.

\section{Supporting Information}

Additional supporting information is attached to the archive record for this article:

Supplementary Figure S1. Geographic locations in the southeastern U.S. from which Schizocosa crassipes were collected in 2016 and/or 2017 for use in laboratory mating trials.

Supplemental Figure S2. Between-year variation in RC scores for the four locations that were sampled in both 2016 and 2017.

Supplemental Table S1. Selection gradients estimated from multiple logistic regression of copulation success on 11 male phenotypic traits.

Supplementary Table S2. Results of AICc model selection to evaluate support for differences among localities in the intercept or slope of the relationship between copulation success and each standardized male phenotypic trait. 
Supplemental Table S3. Results of ANOVAs comparing ranked values of 11 phenotypic traits across collection sites and collection years.

Supplemental Figure S3. Geographic variation in 11 male phenotypic traits.

Supplemental Table S4. Loadings of 11 male phenotypic traits on three rotated components obtained from principal components analysis with varimax rotation.

Supplemental Table S5. Results of AICc model selection to evaluate support for differences among localities in the intercept or slope of the relationship between copulation success and each standardized rotated component (RC) representing male traits.

Supplemental Table S6. Selection gradients estimated from multiple logistic regression of copulation success on 11 male phenotypic traits, but in which male mass and tibial brush area are standardized relative to male cephalothorax width.

Supplementary Table S7. Results of AICc model selection to evaluate support for differences among localities in the intercept or slope of the relationship between copulation success and each standardized male phenotypic trait.

Supplemental Figure S4. Geographic variation in rotated component (RC) scores representing male traits.

Supplemental Figure $\mathbf{S}$. Variation in relative mass (left) and relative tibial brush area (right) among collection sites.

Video S1 


\section{Collection Sites Sampled}

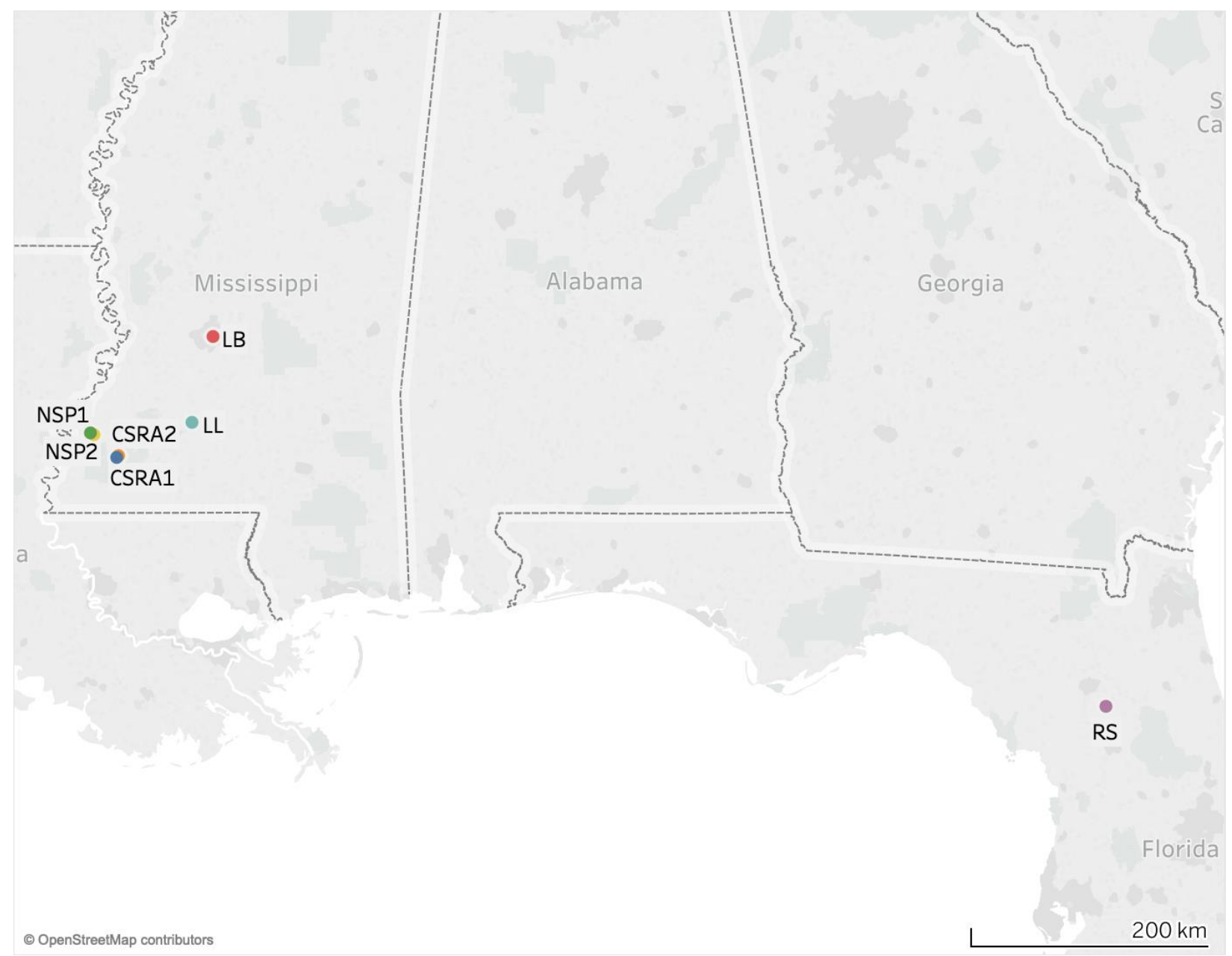

Supplementary Figure S1. Geographic locations in the southeastern U.S. from which Schizocosa crassipes were collected in 2016 and/or 2017 for use in laboratory mating trials. 

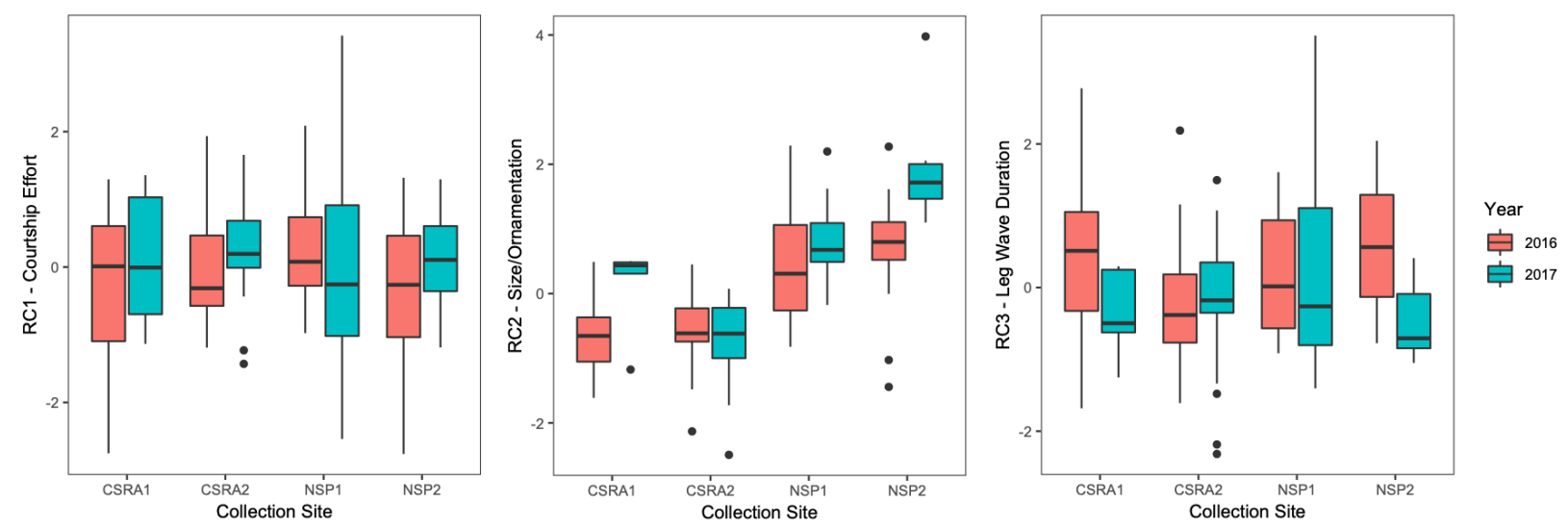

Supplemental Figure S2. Between-year variation in RC scores for the four locations that were sampled in both 2016 and 2017.

\section{Analyses of Individual Traits}

\section{Quantifying Sexual Selection}

In our primary analysis, we used principal components analysis (PCA) to reduce the 11 phenotypic traits we measured into 3 rotated principal components (RCs). We did this to simplify our analysis and to account for correlations among traits that might influence our estimates of their corresponding selection gradients. To determine the extent this approach influences our conclusions, we performed a complementary analysis in which we estimated selection gradients using multiple regression on individual traits (Lande \& Arnold 1983).

We standardized each of the 11 phenotypic traits to their respective site-specific means and standard deviations as was done for principal component scores used in the primary analysis (see main text). We then constructed a binomial logistic regression in which copulation success was modeled as a function of the linear combinations of these standardized phenotype values. We once again used penalized logistic regressions (Firth 1993) using the function brglm() from the R package \{brglm \} (Kosmidis 2017) to minimize issues associated with rare successes or failures among some combinations of predictor variables, and we included collection year in all models. We assessed the significance of the estimated coefficient for each standardized phenotypic trait by comparing the full model to a model excluding the corresponding trait using a likelihood ratio test. To assess the degree to which our estimate of each selection gradient and the associated standard error was influenced by the inclusion of one or more correlated predictors, we also quantified the variance inflation factor (VIF) for each estimate in the model. The VIF estimates the factor by which the estimated variance of a regression coefficient is increased due to the presence of correlated predictors, and therefore provides an indication of the 
caution with which we must interpret the significance of the regression coefficient (O'Brien 2007).

This alternative analysis shows that only the rate at which a male produces body bounces (bounces per minute) predicts copulation success after accounting for the effects of other phenotypic traits (Supplementary Table S1). The VIFs suggest that strong phenotypic correlations may affect the standard errors of the selection gradients for some individual traits. In particular, the total duration of time spent leg-waving (TotalWaveDur) and the log-transformed mean leg-wave bout duration [Log(MeanWaveDur)] have large VIF factors (Supplementary Table S1), suggesting strong correlations with at least one other predictor limit our ability to confidently assess their significance. Unsurprisingly, these two phenotypic measures are highly positively correlated (Estimate \pm S.E. $=0.73 \pm 0.05, F_{1,201}=255.1, P<0.001, R^{2}=0.53$ ).

Excluding either of these two predictors from the model reduces the VIF of the other predictor and results in a significant negative selection gradient [TotalWaveDur: Estimate \pm S.E. $=-0.73 \pm$ 0.33, $\chi_{1}^{2}=5.77, P=0.016, \mathrm{VIF}=3.20 ; \log \left(\right.$ MeanWaveDur): Estimate \pm S.E. $=-0.68 \pm 0.30, \chi_{1}^{2}$ $=6.17, P=0.013, \mathrm{VIF}=2.73 \mathrm{]}$. This does not affect the estimates or significance of other model terms. However, the VIF for each variable when retained in the model remains high, and a negative effect of the total time spent leg waving and the mean duration of leg-waving bouts on copulation success seems biologically counter-intuitive. This suggests that additional among-trait correlations might affect these estimates (O'Brien 2007). Total leg wave duration and mean leg wave bout duration are both highly positively correlated with the number of body bounces per minute (Bounce Rate) [TotalWaveDur: Estimate \pm S.E. $=0.77 \pm 0.05, F_{1,201}=292.6, P<0.001$, $R^{2}=0.59, \log \left(\right.$ MeanWaveDur): Estimate \pm S.E. $=0.46 \pm 0.06, F_{1,201}=52.6, P<0.001, R^{2}=$ $0.21]$. When we assessed a model that eliminated this additional source of collinearity by excluding body bounces per minute as a predictor, neither total leg wave duration (Estimate \pm S.E. $=0.02 \pm 0.23, \chi_{1}^{2}=0.05, P=0.823, \mathrm{VIF}=1.64$ ) nor mean leg wave bout duration (Estimate \pm S.E. $\left.=-0.02 \pm 0.21, \chi_{1}^{2}=0.02, P=0.880, \mathrm{VIF}=1.50\right)$ were significantly associated with copulation success.

\begin{tabular}{lcccccc} 
Trait & Estimate & $\begin{array}{l}\text { Std. } \\
\text { Error }\end{array}$ & $\begin{array}{l}\text { Likelihood } \\
\text { Ratio }\end{array}$ & $\boldsymbol{d f}$ & $\boldsymbol{P}$ & VIF \\
\hline Mass & -0.08 & 0.31 & $<0.01$ & 1 & 0.94 & 2.70 \\
CephWidth & 0.20 & 0.30 & 0.43 & 1 & 0.51 & 2.69 \\
BrushArea & 0.04 & 0.26 & 0.04 & 1 & 0.84 & 2.04 \\
TibialDark & -0.13 & 0.20 & 0.44 & 1 & 0.51 & 1.25 \\
Log(Latency Signal) & 0.13 & 0.25 & 0.25 & 1 & 0.62 & 1.72 \\
Log(Latency Bounce) & -0.28 & 0.29 & 1.14 & 1 & 0.29 & 2.09 \\
Bounce Rate & 1.47 & 0.46 & 12.77 & 1 & $<\mathbf{0 . 0 1}$ & 3.55 \\
Log(MeanBounceInt) & 0.07 & 0.22 & 0.07 & 1 & 0.80 & 1.74 \\
Log(NoWaveBouts) & -0.21 & 0.36 & 0.45 & 1 & 0.50 & 3.87 \\
TotalWaveDur & -0.36 & 0.57 & 0.35 & 1 & 0.56 & 9.19 \\
Log(MeanWaveDur) & -1.06 & 0.51 & 0.75 & 1 & 0.39 & 7.93
\end{tabular}

Supplemental Table S1. Selection gradients estimated from multiple logistic regression of copulation success on 11 male phenotypic traits. Variance inflation factors (VIF) are provided 
for each model term to quantify the degree to which the corresponding estimate of the standard error is inflated due to the inclusion of one or more correlated predictors.

We then repeated the model selection procedure used in our primary analysis to assess the support for differences in selection gradients among collection sites, but using the 11 standardized phenotypic traits instead of the corresponding rotated component scores. The results of this additional model selection procedure corroborate our primary analysis in that the topranked model includes site-specific intercepts, but no interactions between site and the slope of the selection gradient for any of the 11 standardized traits (Supplemental Table S2). This model received about 3 times as much support as the model that contained no variation in intercept or slope of selection gradients among sites (evidence ratio $=0.505 / 0.173=2.92$ ). The only other model that received more support than the model containing no variation in selection gradients was identical to the best model but included site-specific effects of total leg waving duration (TotalWaveDur). However, this model received only marginally more support than the model containing no variation in selection gradients (evidence ratio $=0.253 / 0.173=1.46$ ) and was half as well supported as the best model (evidence ratio 0.253/0.505 $=0.50$ ). In light of the collinearity between total leg waving duration and mean leg waving bout duration that complicated the interpretation of effects in the baseline model (see above), we probed the robustness of the evidence for site-specific effects of total leg waving duration by repeating this model selection procedure for a candidate model set that excluded mean leg waving bout duration from all models. This resulted in identical model rankings, but weaker quantitative support for site-specific effects of total leg waving duration relative to the best model (evidence ratio $=0.210 / 0.595=0.35$ ). When omitting mean leg waving bout duration, the model including site-specific effects of total leg waving duration did not perform significantly better than the topranked model $\left(\chi_{6}^{2}=6.12, P=0.410\right)$.

\section{Quantifying Geographic Variation in Male Display Traits}

To provide more detailed insights into the geographic patterns of phenotypic variation represented by the rotated principal components used in our primary analysis, we also repeated our comparison of phenotypes among locations using the 11 individual phenotypic measures we quantified. The results of the ANOVAs comparing ranked phenotype values among sites (while controlling for collection year and false discovery rate) are shown in Supplemental Table S3. Consistent with the results based on our primary analysis, traits related to male body size (male mass, cephalothorax width, and tibial brush area) differed strongly among collection sites (Supplemental Figure S3). Additionally, the total number of leg waving bouts differed significantly among sites (Supplemental Figure S3), consistent with the marginally nonsignificant geographic variation in RC3 - Leg Wave Duration in our primary analysis (see main text, Figure 3). By focusing on individual traits, we also found significant variation in tibial darkness among collection sites. Because tibial darkness did not load highly on any of the rotated components that explain the majority of the variation in the phenotype data (see main text, Table 2 ), it is not surprising that our primary analysis failed to detect this source of phenotypic variation. Darker forelegs in LL and RS spiders appear to be the main source of this variation (Supplemental Figure S3). Importantly, none of our selection analyses indicated significant selection gradients associated with total leg wave duration or tibial darkness, nor did we find any support for site-specific selection on either of these traits (see above). 


\begin{tabular}{|c|c|c|c|c|c|c|c|c|c|c|c|c|c|c|c|c|}
\hline Site & M:Site & CW:Site & BA:Site & TD:Site & LLS:Site & LLB:Site & BR:Site & LMBI:Site & LNWB:Site & TWD:Site & LMWD:Site & $d f$ & LogLik & $\mathrm{AICc}$ & $\triangle \mathrm{AICc}$ & weight \\
\hline+ & & & & & & & & & & & & 19 & $\begin{array}{l}-79.47 \\
\end{array}$ & 201.1 & 0 & 0.505 \\
\hline \multirow[t]{2}{*}{+} & & & & & & & & & & + & & 25 & -72.56 & 202.5 & 1.38 & 0.253 \\
\hline & & & & & & & & & & & & 13 & -87.66 & 203.2 & 2.15 & 0.173 \\
\hline+ & & & & & & & + & & & & & 25 & -75.08 & 207.5 & 6.42 & 0.020 \\
\hline+ & & & & & & & & + & & & & 25 & -75.61 & 208.6 & 7.48 & 0.012 \\
\hline+ & & & & & & & & & & & + & 25 & -76.12 & 209.6 & 8.49 & 0.007 \\
\hline+ & & & & & + & & & & & & & 25 & -76.12 & 209.6 & 8.50 & 0.007 \\
\hline+ & + & & & & & & & & & & & 25 & -76.63 & 210.6 & 9.52 & 0.004 \\
\hline
\end{tabular}

Supplementary Table S2. Results of AICc model selection to evaluate support for differences among localities in the intercept or slope of the relationship between copulation success and each standardized male phenotypic trait. Each row corresponds to candidate model, and + indicates the inclusion of a term in the model. Each model also included an intercept and the linear effects of each phenotypic trait (see Table S1). Only those models comprising the first $98 \%$ of the cumulative AICc weight are shown. Traits are abbreviated as follows: $M=$ Mass, $C W=$ Cephalothorax Width, $B A=$ Tibial Brush Area, $T D=$ Tibial Darkness, $L L S=\log (\operatorname{Latency}$ to Signal $), L L B=$ $\log ($ Latency to Bounce), $B R=$ Bounces/minute, $L M B I=\log ($ Mean Bounce Interval), $L N W B=\log ($ Number of Leg Waving Bouts), $T W D=$ Total Leg Waving Duration, $L M W D=\log ($ Mean Leg Waving Bout Duration). 


\begin{tabular}{|c|c|c|c|c|}
\hline Trait & Term & $\boldsymbol{F}$ & $d f$ & $P($ adj. $)$ \\
\hline \multirow[t]{2}{*}{ Mass } & Collection Site & 27.74 & 6,195 & $<0.001$ \\
\hline & Year & 9.91 & 1,195 & 0.021 \\
\hline \multirow[t]{2}{*}{ CephWidth } & Collection Site & 27.32 & 6,195 & $<0.001$ \\
\hline & Year & 3.45 & 1,195 & 0.120 \\
\hline \multirow[t]{2}{*}{ BrushArea } & Collection Site & 12.94 & 6,195 & $<0.001$ \\
\hline & Year & 6.81 & 1,195 & 0.054 \\
\hline \multirow[t]{2}{*}{ TibialDark } & Collection Site & 4.93 & 6,195 & $<0.001$ \\
\hline & Year & 4.50 & 1,195 & 0.086 \\
\hline \multirow[t]{2}{*}{ Log(Latency Signal $)$} & Collection Site & 0.66 & 6,195 & 0.686 \\
\hline & Year & 0.37 & 1,195 & 0.665 \\
\hline \multirow{2}{*}{ Log(Latency Bounce) } & Collection Site & 0.75 & 6,195 & 0.672 \\
\hline & Year & 1.21 & 1,195 & 0.375 \\
\hline \multirow[t]{2}{*}{ BounceRate } & Collection Site & 1.41 & 6,195 & 0.290 \\
\hline & Year & 0.18 & 1,195 & 0.742 \\
\hline \multirow[t]{2}{*}{ Log(MeanBounceInt) } & Collection Site & 2.33 & 6,195 & 0.062 \\
\hline & Year & 1.27 & 1,195 & 0.375 \\
\hline \multirow[t]{2}{*}{ Log(NoWaveBouts) } & Collection Site & 2.94 & 6,195 & 0.020 \\
\hline & Year & 4.32 & 1,195 & 0.086 \\
\hline \multirow[t]{2}{*}{ TotalWaveDur } & Collection Site & 0.75 & 6,195 & 0.672 \\
\hline & Year & 0.02 & 1,195 & 0.893 \\
\hline \multirow[t]{2}{*}{ Log(MeanWaveDur) } & Collection Site & 2.03 & 6,195 & 0.099 \\
\hline & Year & 4.76 & 1,195 & 0.086 \\
\hline
\end{tabular}

Supplemental Table S3. Results of ANOVAs comparing ranked values of 11 phenotypic traits across collection sites and collection years. Bolded values are significant at the $\alpha=0.05$ level after correction for false discovery rate. 

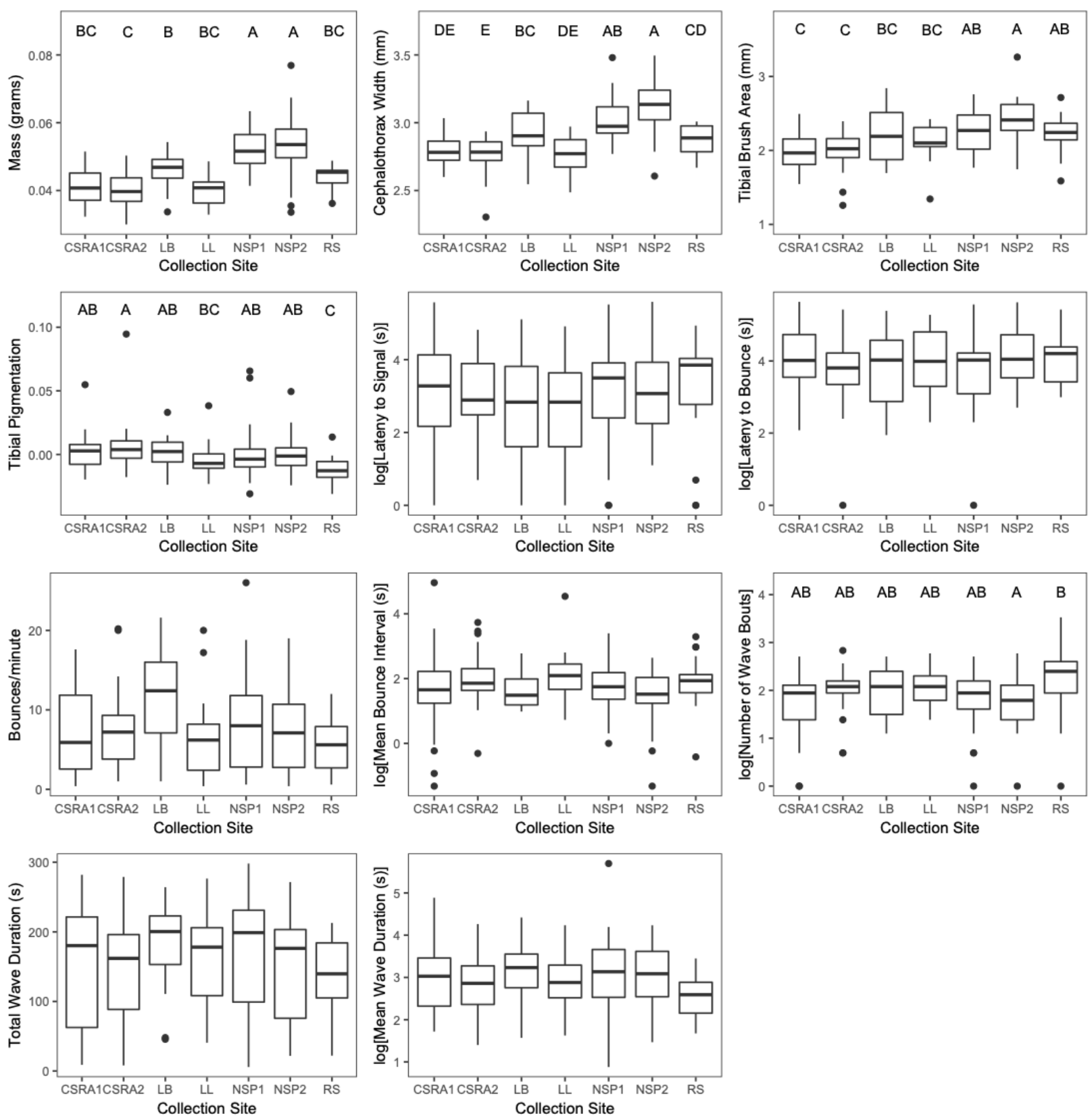

Supplemental Figure S3. Geographic variation in 11 male phenotypic traits. Note that tibial pigmentation values are calculated relative to a white standard such that lower values of tibial pigmentation indicate darker tibiae. Sites that do not share a letter grouping differ significantly from each other according to a Tukey HSD post-hoc analysis. 


\section{Summary}

An alternative analysis using all 11 phenotypic traits rather than 3 rotated principal components results in some ambiguity regarding selection on leg waving behavior due to the high degree of correlation between our two measures of leg waving duration and between these two measures and body bounce rate. Nevertheless, consistent with the interpretation of our primary analysis using principal component scores, body bounce rate was the strongest predictor of copulation success, and under no circumstances did we find support for selection on male morphological traits. Additionally, while assessing the evidence for site-specific selection using all 11 traits resulted in somewhat greater model selection uncertainty, the model including only site-specific intercepts was at least twice as well supported as any alternative model, and support for this model was greater when excluding strongly correlated phenotypic measures from the candidate model set. Finally, comparing all 11 phenotypic measures among collection sites provides additional insight into the variation in leg waving behavior hinted at by our primary analysis and suggests there may also be geographic variation in tibial darkness. Nevertheless, our conclusions remained unchanged - those traits that vary among locations are consistently unrelated to mating success. This is in direct contrast to our prediction (P3) that traits under phenotypic selection through mate choice should differ among locations.

\section{Analysis of Relative Measures of Body Mass and Tibial Brush Area}

\section{Quantifying Sexual Selection}

To account for the possibility that mating success does not depend on the absolute mass or tibial brush area of a male, but rather on how these traits relate to his overall body size, we also performed the multiple regression analysis and subsequent model selection procedure using measures of male mass and tibial brush area relative to their cephalothorax width. The relationship between male mass and cephalothorax width did not differ among sites (Cephalothorax Width*Site interaction: $F_{6,189}=1.44, P=0.203$ ), nor did the relationship between tibial brush area and cephalothorax width (Cephalothorax Width*Site interaction: $F_{6,189}$ $=0.40, P=0.217$ ). Thus, by quantifying relative mass and relative tibial brush area as the residuals of regressions of each trait on cephalothorax width across all males in our study, we created residual mass and residual brush area indices that could be compared among collection sites. Repeating the PCA of male phenotypic traits (see Methods) using residual mass and residual tibial brush area resulted in somewhat different loadings of the phenotype RCs (Supplemental Table S4). Similar to our primary analysis based on raw measures of mass and brush area, RC1 represented variation in overall courtship effort as reflected by the high loadings of $\log$ (Latency to Signal), $\log$ (Latency to Bounce), Bounce Rate, Total Leg Wave Duration and Mean Leg Waving Bout Duration. In contrast to the primary analysis, however, RC2 rather than RC3 represented variation in courtship behavior not explained by overall courtship effort (i.e., individuals with fewer leg waving bouts, longer average leg waving bout durations, and shorter intervals between body bounces). RC3 was instead most strongly associated with Tibial Darkness, although Cephalothorax Width and Relative Tuft area also load somewhat highly on this component. For the remainder of this section, we refer to these RCs as 'Courtship Effort', 
'Leg Wave Duration', and 'Size/Ornamentation' to facilitate comparison to our primary analysis, though the precise loadings of the individual traits on these RCs differ to some degree (cf. Supplemental Table S4, Table 2).

\begin{tabular}{|lccc|}
\multicolumn{1}{c}{} & $\begin{array}{l}\text { RC1 }- \\
\text { Courtship Effort }\end{array}$ & $\begin{array}{l}\text { RC2 } \\
\text { Leg Wave Duration }\end{array}$ & \multicolumn{2}{c}{$\begin{array}{l}\text { RC3 }- \\
\text { Size/Ornamentation }\end{array}$} \\
\hline Mass & 0.00 & 0.05 & -0.14 \\
CephWidth & 0.02 & 0.18 & 0.42 \\
BrushArea & 0.10 & -0.17 & 0.48 \\
TibialDark & 0.03 & 0.25 & $\mathbf{- 0 . 8 1}$ \\
LatencySignal & $\mathbf{- 0 . 7 0}$ & 0.30 & 0.13 \\
LatencyBounce & $\mathbf{- 0 . 8 0}$ & 0.22 & 0.05 \\
BounceRate & $\mathbf{0 . 8 5}$ & 0.21 & -0.01 \\
BounceInt & -0.12 & $\mathbf{- 0 . 6 9}$ & 0.17 \\
NoWaveBouts & 0.34 & $\mathbf{- 0 . 7 9}$ & -0.13 \\
TotalWaveDur & $\mathbf{0 . 9 0}$ & 0.15 & 0.17 \\
MeanWaveDur & $\mathbf{0 . 5 8}$ & $\mathbf{0 . 5 6}$ & 0.32 \\
\hline Prop. Variance & 0.29 & 0.16 & 0.12 \\
Cum. Variance & 0.29 & 0.45 & 0.56
\end{tabular}

Supplemental Table S4. Loadings of 11 male phenotypic traits on three rotated components obtained from principal components analysis with varimax rotation. Axes are re-labeled according to those phenotypic traits which loaded most highly on the component. Bolded values represent for each component those traits with loadings greater than 0.50 in absolute magnitude.

We once again found significant effects of Courtship Effort $\left(\chi_{1}^{2}=9.50, P=0.002\right)$, but not Size/Ornamentation $\left(\chi_{1}^{2}=0.17, P=0.680\right)$ or Leg Wave Duration $\left(\chi_{1}^{2}=0.04, P=0.838\right)$, on male copulation success (overall model $\chi_{3}^{2}=9.93, P=0.019$ ). Males with greater Courtship Effort scores were more likely to copulate (estimate \pm std. error $=0.537 \pm 0.185$, approx. $\beta=$ $0.087 \pm 0.030)$. However, males with greater Size/Ornamentation scores were not significantly more likely to copulate (estimate \pm std. error $=0.073 \pm 0.168$, approx. $\beta=0.012 \pm 0.028$ ), nor were males with greater Leg Wave Duration scores (estimate \pm std. error $=0.035 \pm 0.170$, approx. $\beta=0.006 \pm 0.029$ ).

Consistent with our primary analysis, there was little support for statistical models including site-specific effects of the RCs representing male traits, though there was support for different intercepts among sites (Supplemental Table S5). The best-supported model, which included site-specific intercepts, received about 4.5 times more support than the next bestsupported model, which included no variation in slopes or intercepts of the relationships between male trait RCs and copulation success (evidence ratio $=0.767 / 0.174=4.41$ ). No other candidate 
model representing variation in phenotypic selection among localities received more support than the model including no variation in slopes or intercepts (i.e., all evidence ratios $\geq 3$ in support of the latter) (Supplemental Table S5).

\begin{tabular}{|c|c|c|c|c|c|c|c|c|}
\hline Site & Courtship Effort:Site & Size/Ornamentation:Site & LegWaveDuration:Site & $d f$ & LogLik & AICc & $\Delta \mathrm{AICc}$ & weight \\
\hline \multirow[t]{2}{*}{+} & & & & 11 & -91.56 & 206.5 & 0 & 0.767 \\
\hline & & & & 5 & -99.58 & 209.5 & 2.97 & 0.174 \\
\hline+ & + & & & 17 & -87.30 & 211.9 & 5.41 & 0.051 \\
\hline+ & & & + & 17 & -89.63 & 216.6 & 10.06 & 0.005 \\
\hline+ & & + & & 17 & -90.41 & 218.1 & 11.62 & 0.002 \\
\hline+ & + & & + & 23 & -85.73 & 223.6 & 17.12 & $<0.001$ \\
\hline+ & + & + & & 23 & -86.67 & 225.5 & 19.01 & $<0.001$ \\
\hline+ & & + & + & 23 & -89.40 & 231.0 & 24.46 & $<0.001$ \\
\hline+ & + & + & + & 29 & -86.18 & 240.4 & 33.91 & $<0.001$ \\
\hline
\end{tabular}

Supplemental Table S5. Results of AICc model selection to evaluate support for differences among localities in the intercept or slope of the relationship between copulation success and each standardized rotated component (RC) representing male traits. Each row corresponds to candidate model, and + indicates the inclusion of a term in the model. Each model also included an intercept and the linear effects of each rotated component. Standardized rotated components are referred to by the labels described in Supplemental Table S3.

Substituting these relative measures of mass and tibial brush area in the multiple regression analysis based on all 11 individual traits (see Individual Traits - Quantifying Female Mate Choice Behavior) resulted in lower VIFs for male morphological traits but did not affect the conclusions of the analysis (Supplemental Table S6). Similarly, substituting these relative measures of mass and tibial brush area into the candidate models used to assess the support for site differences in selection using did not affect the qualitative model rankings and resulted in nearly identical model weights (cf. Supplemental Table S2, Supplemental Table S7).

\begin{tabular}{|c|c|c|c|c|c|c|}
\hline Trait & Estimate & $\begin{array}{l}\text { Std. } \\
\text { Error }\end{array}$ & $\begin{array}{l}\text { Likelihood } \\
\text { Ratio }\end{array}$ & $d f$ & $\boldsymbol{P}$ & VIF \\
\hline Relative Mass & -0.12 & 0.21 & 0.25 & 1 & 0.62 & 1.19 \\
\hline CephWidth & 0.14 & 0.19 & 0.58 & 1 & 0.45 & 1.10 \\
\hline Relative BrushArea & 0.07 & 0.21 & 0.07 & 1 & 0.80 & 1.26 \\
\hline TibialDark & -0.11 & 0.20 & 0.29 & 1 & 0.59 & 1.27 \\
\hline Log(Latency Signal) & 0.12 & 0.24 & 0.22 & 1 & 0.64 & 1.70 \\
\hline Log(Latency Bounce) & -0.27 & 0.29 & 1.10 & 1 & 0.29 & 2.07 \\
\hline
\end{tabular}




$\begin{array}{lcccccc}\text { Bounce Rate } & 1.47 & 0.46 & 12.87 & 1 & <\mathbf{0 . 0 1} & 3.54 \\ \text { Log(MeanBounceInt) } & 0.07 & 0.22 & 0.09 & 1 & 0.77 & 1.75 \\ \text { Log(NoWaveBouts) } & -0.20 & 0.36 & 0.44 & 1 & 0.51 & 3.87 \\ \text { TotalWaveDur } & -0.41 & 0.56 & 0.38 & 1 & 0.54 & 9.05 \\ \text { Log(MeanWaveDur) } & -1.06 & 0.51 & 0.76 & 1 & 0.39 & 7.85\end{array}$

Supplemental Table S6. Selection gradients estimated from multiple logistic regression of copulation success on 11 male phenotypic traits, but in which male mass and tibial brush area are standardized relative to male cephalothorax width. Variance inflation factors (VIF) are provided for each model term to quantify the degree to which the corresponding estimate of the standard error is inflated due to the inclusion of one or more correlated predictors. 


\begin{tabular}{|c|c|c|c|c|c|c|c|c|c|c|c|c|c|c|c|c|}
\hline Site & RM:Site & CW:Site & RBA:Site & TD:Site & LLS:Site & LLB:Site & BR:Site & LMBI:Site & LNWB:Site & TWD:Site & LMWD:Site & $d f$ & LogLik & AICc & $\triangle \mathrm{AICc}$ & weight \\
\hline+ & & & & & & & & & & & & 19 & $\begin{array}{l}-79.34 \\
\end{array}$ & 200.8 & 0 & 0.476 \\
\hline+ & & & & & & & & & & + & & 25 & -72.26 & 201.9 & 1.02 & 0.285 \\
\hline & & & & & & & & & & & & 13 & -87.51 & 203.0 & 2.12 & 0.165 \\
\hline+ & & & & & & & + & & & & & 25 & -74.76 & 206.9 & 6.04 & 0.023 \\
\hline+ & & & & & & & & + & & & & 25 & -75.33 & 208.0 & 7.18 & 0.013 \\
\hline+ & & & & & & & & & & & + & 25 & -75.84 & 209.0 & 8.19 & 0.008 \\
\hline+ & & & & & + & & & & & & & 25 & -76.03 & 209.4 & 8.57 & 0.007 \\
\hline+ & + & & & & & & & & & & & 25 & -76.74 & 210.8 & 10.00 & 0.003 \\
\hline
\end{tabular}

Supplementary Table S7. Results of AICc model selection to evaluate support for differences among localities in the intercept or slope of the relationship between copulation success and each standardized male phenotypic trait. Each row corresponds to candidate model, and + indicates the inclusion of a term in the model. Each model also included an intercept and the linear effects of each phenotypic trait. Only those models comprising the first $98 \%$ of the cumulative AICc weight are shown. Traits are abbreviated as follows: $R M=$ Relative Mass, $C W=$ Cephalothorax Width, $R B A=$ Relative Tibial Brush Area, $T D=$ Tibial Darkness, $L L S=\log (\operatorname{Latency}$ to Signal), $L L B=\log ($ Latency to Bounce $), B R=$ Bounces/minute, $L M B I=\log ($ Mean Bounce Interval $), L N W B=\log ($ Number of $\operatorname{Leg} W a v i n g$ Bouts), $T W D=$ Total Leg Waving Duration, $L M W D=\log ($ Mean Leg Waving Bout Duration). 


\section{Quantifying Geographic Variation in Male Display Traits}

When we used relative mass and relative tibial brush area in our PCA analysis, we found geographic variation in both Size/Ornamentation $\left(F_{6,195}=6.03, P<0.001\right)$ and Leg Wave Duration $\left(F_{6,195}=6.12, P<0.001\right)$, but not Courtship Effort $\left(F_{6,195}=0.79, P=0.58\right)$ (Supplemental Figure S4). Thus, using relative mass and relative tibial brush produces a RC associated with leg-waving behavior that varies more strongly across collection sites, yet these patterns still contrast our prediction (P3) that traits under phenotypic selection through mate choice should differ among locations.
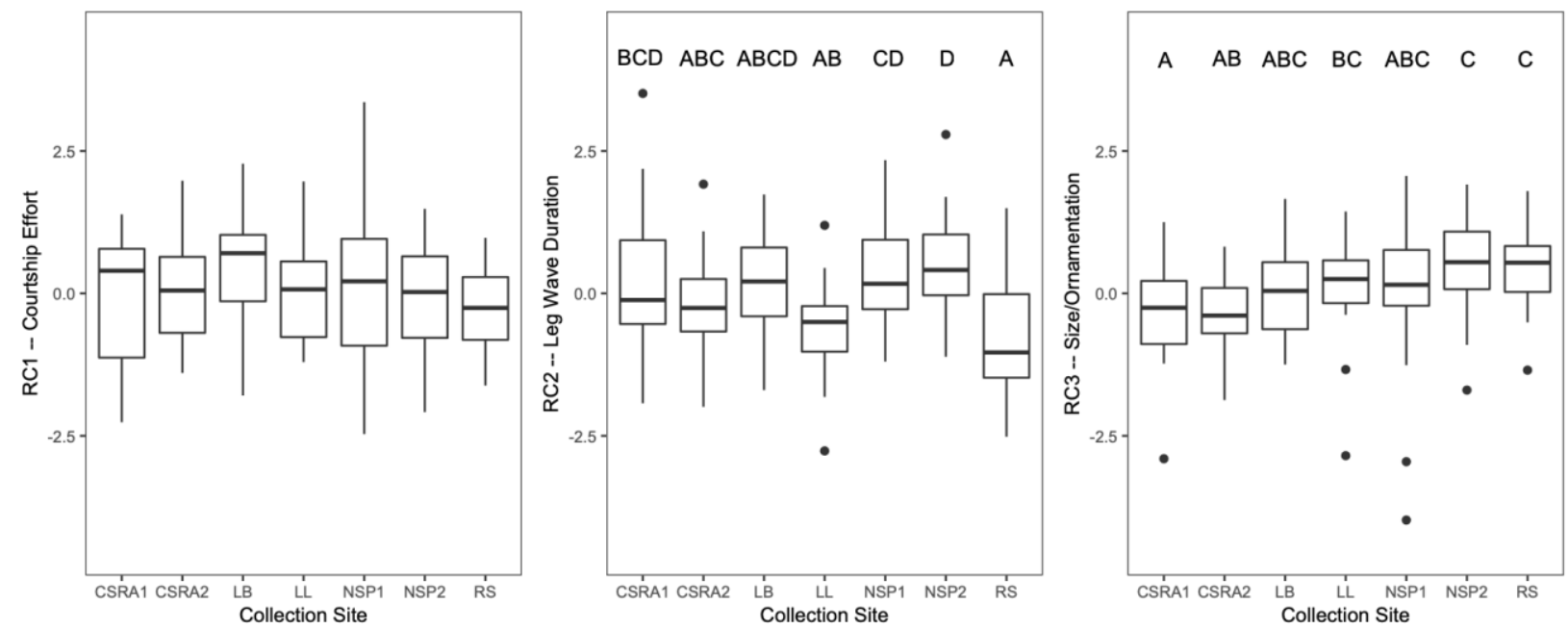

Supplemental Figure S4. Geographic variation in rotated component (RC) scores representing male traits. Sites that do not share a letter grouping differ significantly from each other according to a Tukey HSD post-hoc analysis.

After controlling for differences between years, relative mass $\left(F_{6,195}=4.26, P<0.001\right)$ and relative tibial brush area $\left(F_{6,195}=3.29, P=0.004\right)$ differed among collection sites (Supplemental Figure S5), indicating that variation in these traits among sites exceeds what would be expected based on differences in body size alone. For relative mass, only one site differed from any others: males from NSP1 were significantly more massive relative to their body size than males from CSRA1, CSRA2, LL, or LB (Tukey HSD, all $P<0.05$ ), but not males from NSP2 or RS. Relative tibial brush area was greater in LL than CSRA1 (Tukey HSD, $P=$ 0.003), but no other pairwise comparisons were significant. 

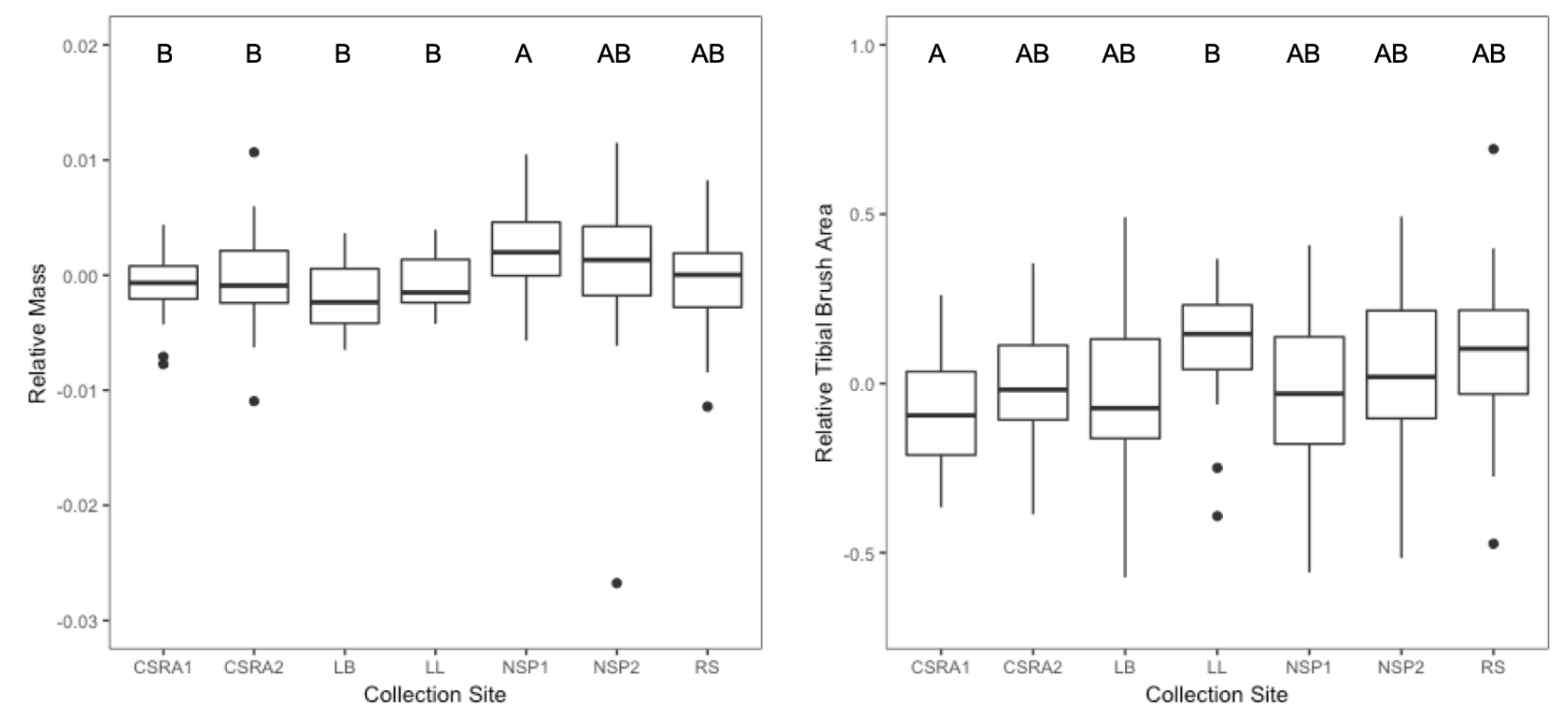

Supplemental Figure S5. Variation in relative mass (left) and relative tibial brush area (right) among collection sites. Sites that do not share a letter grouping differ significantly from each other according to a Tukey HSD post-hoc analysis. 


\section{References}

Firth, D. 1993. Bias reduction of maximum likelihood estimates. Biometrika 80:27-38.

Lande, R. and Arnold, S. J. 1983. The measurement of selection on correlated characters. Evolution 37:1210-1226.

Kosmidis, I. 2017. brglm: Bias Reduction in Binary-Response Generalized Linear Models. R package version 0.6.1. <URL: http://www.ucl.ac.uk/ ucakiko/software.html>.

O'Brien, R. M. 2007. A caution regarding rules of thumb for variance inflation factors. Quality \& quantity 41:673-690. 